\title{
Trajectory Control for Very Flexible Aircraft
}

\author{
Christopher M. Shearer* and Carlos E. S. Cesnik亡 \\ University of Michigan, Ann Arbor, Michigan 48109 \\ DOI: $10.2514 / 1.29335$
}

\begin{abstract}
This paper focuses on trajectory control of the six-degree-of-freedom body fixed reference frame located on a very flexible aircraft. The six-degree-of-freedom equations of motion of a reference point on the aircraft are coupled with a low-order strain-based nonlinear structural analysis and an unsteady finite state potential flow aerodynamics model. Because of the inherent flexibility of the aircraft, the low-order structural frequencies are of the same order as the rigid-body mode frequencies. This coupling is accounted for in the controller development. A heuristic approach based upon pilot behavior is developed. The approach separates the problem into two parts: a fast inner loop and a slower outer loop. Dominant kinematic nonlinearities are handled in the outer loop, whereas the inner loop is further separated into a lateral and longitudinal motion. Control of the inner-loop lateral motion is accomplished using a standard linear quadratic regulator. For the longitudinal motion, dynamic inversion is used. Differences between the desired and actual trajectories are handled using a nonlinear proportional, integral, derivative approach. The closed-loop time integration is accomplished using an implicit modified Newmark method. A capstone numerical simulation is presented, highlighting the strengths and weaknesses of the method.
\end{abstract}

\begin{tabular}{|c|c|}
\hline \multicolumn{2}{|r|}{ Nomenclature } \\
\hline$A, B, C$ & $=$ linear state-space matrices \\
\hline$B$ & $\begin{aligned} &= \text { body fixed reference frame; distributed and } \\
& \text { point control input influence matrices }\end{aligned}$ \\
\hline$b$ & $\begin{aligned}= & \text { displacements and rotations as time integral of } \\
& \beta ; \text { semichord length }\end{aligned}$ \\
\hline$C_{L_{\alpha}}$ & $=$ coefficient of lift \\
\hline$C^{G B}$ & $=$ rotation matrix from $B$ and $G$ frame \\
\hline$e$ & $=$ error state \\
\hline$F$ & $=$ applied force \\
\hline $\mathbf{F}_{B}$ & $=$ linear force vector applied at $B$ reference frame \\
\hline$F_{i}$ & $\begin{array}{l}=\text { inflow state matrices for inflow differential } \\
\text { equation }\end{array}$ \\
\hline$f(\cdot)$ & $=$ generic function \\
\hline$f_{\mathrm{dst}}, f_{\mathrm{pt}}$ & $=$ distributed and point forces \\
\hline$G$ & $=$ inertial frame \\
\hline$g$ & $\begin{array}{l}=\text { nonlinear state-dependent control influence } \\
\text { vector function }\end{array}$ \\
\hline$g_{0}$ & $=$ gravity column vector \\
\hline$H$ & $=$ modified Jacobian matrix \\
\hline$h$ & $\begin{array}{l}=\text { position and orientation column vector of } \\
\text { flexible structure; rate of climb }\end{array}$ \\
\hline$I$ & $=$ identity matrix \\
\hline $\mathbf{I}_{B}$ & $\begin{array}{l}=\text { inertia dyadic with respect to the } B \text { reference } \\
\text { frame }\end{array}$ \\
\hline$I_{B}$ & $=$ inertia matrix \\
\hline$J$ & $=$ Jacobian matrix \\
\hline$J_{\text {trim }}$ & $=$ trim cost function \\
\hline$K$ & $=$ closed-loop gain matrix or function \\
\hline
\end{tabular}

Presented as Paper 6316 at the AIAA Guidance, Navigation, and Control Conference and Exhibit, Keystone, CO, 21-24 August 2006; received 16 December 2006; revision received 17 July 2007; accepted for publication 19 July 2007. Copyright $@ 2007$ by Christopher M. Shearer and Carlos E. S. Cesnik. Published by the American Institute of Aeronautics and Astronautics, Inc., with permission. Copies of this paper may be made for personal or internal use, on condition that the copier pay the $\$ 10.00$ per-copy fee to the Copyright Clearance Center, Inc., 222 Rosewood Drive, Danvers, MA 01923; include the code 0731-5090/08 $\$ 10.00$ in correspondence with the CCC.

${ }^{*}$ Major, U.S. Air Force, Ph.D. Candidate; currently Assistant Professor of Aerospace Engineering, Air Force Institute of Technology; christopher. shearer@afit.edu. Student Member AIAA.

${ }^{\dagger}$ Associate Professor of Aerospace Engineering; cesnik@umich.edu. Associate Fellow AIAA. $k_{x}, k_{d x}, k_{I x}, \quad=$

$k_{I I x}, k_{I I I x}$

$L^{\text {aero }}, L^{\delta}$

$M$

$M, C, K$

$\mathbf{M}_{B}$

$M_{\mathrm{dst}}, M_{\mathrm{pt}}$
$M^{\text {aero }}, M^{\delta}$

$m$

$n_{\text {load }}$

O

$P$

$p$

$p_{a}$

$\mathbf{p}_{B}$

$\mathbf{p}_{B}$
$p_{B}$

$p_{r}$

$\mathbf{p}_{r_{\mathrm{c}, \mathrm{m}}}$

$Q$

$Q_{M}$

$q$

$R$

$R_{F}$

$R_{B}$

S

$\mathbf{S}$

$s$

$T$

$t$

$u$

V
$=$ member index, a member is a collection of elements

$=$ proportional, derivative, integral, double integral, and triple integral gains of a given state $x$

$=$ airfoil lift force and lift force due to control surface deflection, respectively

$=$ applied moment

$=$ generalized mass, damping, and stiffness matrices

$=$ moment vector applied at $B$ reference frame

$=$ distributed and point moments

$=$ airfoil pitching moment and pitching moment because of control surface deflection, respectively

$=$ mass per unit span

$=$ normal acceleration

$=$ origin of $B$ reference frame

$=$ aircraft roll rate

$=$ position of the origin of the $w$ frame with respect to the origin of the inertial frame

$=$ position of an arbitrary point in the vehicle with respect to origin of the inertial frame

$=$ inertial position vector of the $B$ reference frame

$=$ inertial position of $B$ reference frame

$=$ position from $B$ reference frame origin to local $w$ reference frame

$=$ vector from $B$ reference frame origin to the center of mass

$=$ aircraft pitch rate; state weighting matrix used in controller design

$=$ portion of generalized mass matrix inverse

$=$ generalized displacement column vector

$=$ generalized force column vector; aircraft yaw rate; control input weighting matrix used in controller design

$=$ generalized force applied to elastic states

$=$ generalized force applied to $B$ reference frame states

$=$ wing area

$=$ search variable column vector

$=$ undeformed beam spatial coordinate

$=$ transpose operator

$=$ time

$=$ aircraft longitudinal velocity; control input

$=$ total aircraft velocity 


$\begin{array}{lll}v & =\text { aircraft lateral velocity } \\ v_{B} & =\text { linear velocity of } B \text { reference frame } \\ w & & \text { local elastic reference frame; aircraft vertical } \\ w_{x}, w_{y}, w_{z} & =\text { column vectors of unit vector components of the } \\ & & \text { local elastic reference frame } \\ x & = & \text { generic state-space variable } \\ y & = & \text { angle of attack } \\ \alpha & = & \text { column vector of } B \text { reference frame linear and } \\ \beta & = & \text { angular velocities } \\ & =\text { flight path elevation angle } \\ \gamma & =\text { control surface angle deflection } \\ \Delta & =\text { column vector of elastic strain state } \\ \delta_{u} & =\text { element strains corresponding to extension, } \\ \epsilon & =\text { twist, and in- and out-of-plane bending } \\ \epsilon_{x}, \kappa_{x}, \kappa_{y}, \kappa_{z} & \text { generalized } B \text { reference frame rotation vector } \\ \Theta_{B} & =\text { column vector of inflow states } \\ \lambda & =\text { inflow velocity } \\ \lambda_{0} & =\text { flight path bank angle } \\ \mu & =\text { density } \\ \rho & =\text { quaternion column vector used for } B \text { reference } \\ \zeta & =\text { frame orientation } \\ \Phi, \Theta, \Psi & =\text { Euler aircraft angles of pitch, roll, and yaw } \\ \chi & =\text { state-space linearized variable } \\ \Omega & =\text { matrix of angular velocities } \omega_{B} \\ \omega_{B} & =\text { angular velocity vector of } B \text { reference frame } \\ \omega_{B} & =\text { angular velocity of } B \text { reference frame } \\ * & = & \text { derivative with respect to time } \\ \cdot) & & \end{array}$

Subscripts

$a$

bb, bf

c.m.

com

des

$e$

ext

$F$

$f$

$\mathrm{ff}, \mathrm{fb}$

$\begin{array}{ll}G & =\text { global matrix } \\ h \epsilon, h b, h & =\text { vector with respect to strain, } \epsilon \text {, or displacement/ }\end{array}$

lat. $\quad=$ lateral

long. $\quad=$ longitudinal

$r \quad=$ relative; rudder

$s \quad=$ wing sweep

$t \quad=$ wing twist; thrust

$u \quad=$ control input

$x, y, z=$ reference to $x, y$, and $z$ coordinates

$0 \quad$ initial value

Superscript

aero

$=$ related to aerodynamic effects

\section{Introduction}

$\mathbf{R}$ ECENT advances in airborne sensors and communication packages have brought the need for high-altitude longendurance (HALE) aircraft. These platforms can be categorized under three broad missions, each supporting either the military or civilian communities. The missions include airborne intelligence, surveillance, and reconnaissance (ISR) for the military [1]; network communication nodes for military and civilian usage [2]; and general atmospheric research [2]. Because of the mission requirements, the desired vehicles are characterized by high-aspect-ratio wings and slender fuselages resulting in very flexible vehicles. Examples of mission optimization studies for this class of vehicle can be found in [1], in which it is shown that the aircraft are required to have a fuel fraction greater than $66 \%$. This results in a very small structural weight fraction. The combination of high aerodynamic efficiency and low structural weight fraction yields inherently flexible wings and nonlinear structural and flight dynamics. The HALE vehicle will then be susceptible to large dynamic wing deformations at low frequencies, presenting a direct impact into the flight dynamic characteristics of the vehicle and controller design, as was seen in the Helios flight tests [3].

The HALE aircraft mission will be unmanned because of its "dull, dirty, or dangerous" [4] nature; in other words,

\begin{abstract}
the attributes that make the use of unmanned preferable to manned aircraft ... [are] in the case of the dull, the better sustained alertness of machines over that of humans and, for the dirty and the dangerous, the lower political and human cost if the mission is lost, and greater probability that the mission will be successful. Lower downside risk and higher confidence in mission success are two strong motivators for continued expansion of unmanned aircraft systems.
\end{abstract}

In 1914, Lawerence Sperry, son of Elmer Sperry, demonstrated his father's autopilot over Paris by standing up in the cockpit of his airplane and having his mechanic walk out on the wing to create an external disturbance [5]. Sperry's invention was capable of maintaining pitch, roll, and heading angles [6]. This remarkable demonstration ushered in the use of the autopilot for a variety of aircraft and aircraft missions. Sperry's autopilot relied upon linear techniques and a relatively stiff biplane box type of construction. Since then, control theory has evolved significantly. For an overview of aeroelastic control, Mukhopadhyay [7] provides an excellent review paper of both analysis and control of aeroelastic structures over the past 100 years. He provides a summary of elastic theory, unsteady aerodynamics, and control, and he highlights the future of nonlinear aeroelastic control. Recently, researchers have looked at applying linear, robust control, and nonlinear techniques for aeroelastic control.

Modern linear control techniques such as linear quadratic regulator (LQR) and linear quadratic Gaussian (LQG) observers have been applied by several researchers. Newman and Buttrill [8] have researched the longitudinal flight dynamics, longitudinal linear controller design, and sensor location for a high-speed transport. Their aircraft modeling shows the importance of including elastic modes and sensor location when designing controllers for flexible aircraft and the difficulty in suppressing aeroelastic dynamic effects from the rigid-body response of a flexible supersonic transport. Tuzcu [9] and Meirovitch and Tuzcu [10] have coupled nonlinear rigid-body dynamics with linear structural dynamics. The formulation treats structural dynamics as disturbance inputs to the rigid-body dynamics. Results are presented for lightweight transport aircraft using standard LQR and LQG control theory. Pedro and Bigg [11] have also applied traditional LQR and LQG techniques to a flexible aircraft. They developed a simulation to evaluate pilot ratings for an aeroelastic longitudinal aircraft model. Their approach looks only at the longitudinal dynamics with a Dryden gust model. Cesnik and Ortega-Morales [12] have used the geometrically exact beam formulation, coupled with finite state aerodynamics and imbedded actuation, to study and control flutter of a HALE-type cantilevered wing. Their work focuses on the use of LQG observers to optimize sensor type, sensor placement, and actuation distribution. They have shown that a single strain gauge sensor, optimally placed, can provide the sensing necessary to control flutter of a high-aspectratio cantilevered wing.

Robust techniques using $H_{1}, H_{2}, H_{\infty}$, and $\mu$ synthesis have been studied by several researchers. Chavez and Schmidt [13] used linear structural modes to develop longitudinal controllers based upon $\mu$ 
synthesis. Their work focuses on developing a systematic approach in which robust controllers can be controlled without complete knowledge of the flexible system. They also present techniques for identifying structural modes of interest and methods of incorporating unsteady aerodynamics directly into the robust formulation without the necessity of building an aerodynamic state-space model. Kron et al. [14] have applied a reduced order $H_{\infty}$ controller to a twodegree-of-freedom (DOF) lateral model of highly flexible transport aircraft. Their work focuses on characterizing model uncertainty when reducing the structural model. The uncertainty is then handled systematically through the development of a 2-DOF $H_{\infty}$ lateral controller. Finally, numerical results are presented, validating their method. Li and Agarwal [15] have also studied the use of a reduced order structural modeling to develop a linear robust controller using $\mathrm{H}_{2}$ and $\mathrm{H}_{\infty}$ techniques for a high-speed civil transport aircraft. They have shown, for that class of aircraft, that the model reduction provides sufficient information for the linearized controller. Goman et al. [16] have conducted a parametric study of various $H_{\infty}$-based and traditional LQR/LQG controllers on a longitudinal elastic aircraft model. Their study shows similar controller designs between the two methods and improved robustness using the $H_{\infty}$-based design.

Dardenne and Ferreres [17] have designed a lateral controller for highly flexible transport aircraft. The design assumes a linear time invariant (LTI) plant and uses a linear quadratic/programming procedure to design the controller using frequency domain constraints. Their initial results have looked at reducing the wingbending effects on lateral motion. Patil [18] and Patil and Hodges [19] have also used minimization routines in designing a static output feedback (SOF) controller used for flutter suppression of HALE-type aircraft. The resulting controller is of a much lower order than LQR/ LQG type controllers but is valid only for a single operating condition. The authors use gain scheduling of different SOF controllers to expand the operating conditions.

Calise et al. [20] have studied the use of output feedback (dynamic inversion) mixed with a neural network plant perturbation estimate to control a longitudinal flexible aircraft model. The process assumes a nonminimum phase (NMP) system with stable zero dynamics. Calise et al. [20] present initial results showing that their method could suppress structural mode interaction for a simplified longitudinal aircraft model. Krishnaswamy and Bugajski [21] have used dynamic inversion for studying control of rockets with fuel slosh. A key aspect of their study is the use of an underactuated system. They have developed an observer model for estimating the fuel slosh dynamics, showing that the resulting controller can control the pitch dynamics of the booster vehicle while damping fuel slosh dynamics. Finally, Gregory [22-25] has applied a novel dynamic inversion control technique for suppressing longitudinal motion because of linear aeroelastic effects of high-speed transports. The variation in the dynamic inversion is the inclusion of prefilters to move the structural modes further into the left-half plane. Results show the ability to significantly improve ride control and longitudinal aircraft handling in the presence of longitudinal structural modes.

Although most of the aeroelastic control work summarized here deals with different classes of aeroelastic effects than the very flexible HALE-type aircraft, the control techniques, both linear and nonlinear, have potential relevance to trajectory control of very flexible aircraft. This paper presents an initial control architecture development proposed for trajectory control of the 6-DOF body fixed reference frame located on a very flexible aircraft. The nonlinear 6-DOF equations of motion of a reference point are coupled with a low-order strain-based nonlinear structural analysis and an unsteady finite state potential flow aerodynamics model. Previously published work on aeroelastic control has centered on aircraft in which the elastic modes are either separated by an order of magnitude or more from the rigid-body modes, or linear elastic theory has been sufficient to describe the behavior of the flexible structure. Although certain limiting assumptions are made (i.e., full elastic and rigid-body state feedback, perfect modeling, and so on), the paper is intended to be a first step in the development of controllers for very flexible aircraft.

\section{Theoretical Formulation}

There are two fundamental approaches when developing a controller. The first is to treat the system of interest as a black box in which the physics of the box are not known, but rather linearized transfer functions can be fit to input/output relationships. The second approach is to start with the known physics of the model and develop the controller. The later is used here and hence requires a basic understanding of the underlying governing differential equations.

The objective of the controller is to provide closed-loop reference tracking of a body fixed reference frame $B$ at point $O$, which in general is not the aircraft's center of mass (Fig. 1), while including the effects of nonlinear aeroelasticity. Although arbitrary, the $B$ reference frame is chosen to be at a convenient location on the elastic aircraft in which linear and angular velocities are tracked (e.g., the location of an inertial measuring unit). Elastic members are then modeled as beams that propagate from the $B$ reference frame origin $O$ or with rigid offsets from the origin. Typically, the $y$ axis is chosen to be tangent to the undeformed longitudinal axis of the fuselage. The $x$ axis is chosen to be positive out the right wing. In this way, the undeformed aircraft planform will be on a plane parallel to the $x-y$ plane defined by the inertial frame $G$. The $z$ axis is simply the cross product of the $x$ and $y$ axes. The tracking will consist of maintaining desired linear and angular velocities of the $B$ reference frame. The means for propagating the reference frame $B$, forward in time is done by deriving and integrating a series of first-order differential equations of the form

$$
\dot{x}=f(x, u)
$$

where $x$ represents the states of the reference frame $B$, and $u$ represents control surface and external inputs. Depending on the fidelity of the analysis, these first-order differential equations vary in their complexity from simple linear time invariant to nonlinear timevarying differential equations. For the classic rigid-body analysis [26], the first-order differential equations take the form

$$
\begin{gathered}
\dot{v}_{B}=f_{v_{B}}\left(v_{B}, \omega_{B}, \zeta, p_{B}, g_{0}, m, F_{\mathrm{ext}}\right) \\
\dot{\omega}_{B}=f_{\omega_{B}}\left(\omega_{B}, I_{B}, \zeta, p_{B}, M_{\mathrm{ext}}\right) \quad \dot{\zeta}=f_{\zeta}\left(\omega_{B}, \zeta\right) \\
\dot{p}_{B}=f_{p_{B}}\left(\zeta, v_{B}\right)
\end{gathered}
$$

where the $B$ reference frame linear and angular velocity variables are represented by $v_{B}$ and $\omega_{B} ; F_{\text {ext }}$ and $M_{\text {ext }}$ are, in general, statedependent external forces and moments; $m$ is the aircraft mass, and $I_{B}$ is the aircraft's inertia matrix about the origin of the $B$ reference frame. The orientation of the $B$ reference frame is accomplished in a variety of ways, from a minimum representation using three nonorthogonal Euler angles to nonminimum four-parameter quaternion representation to a nine-parameter set corresponding to

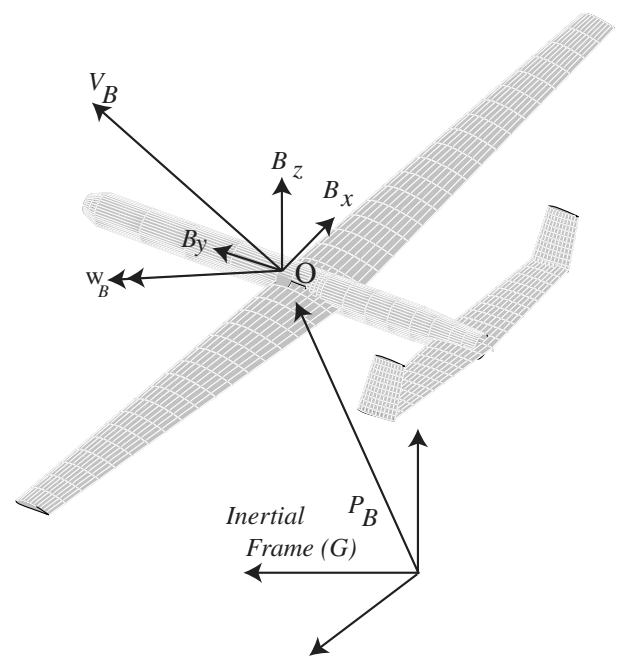

Fig. 1 Basic body reference frame and vehicle coordinates. 
the nine components of the set of unit vectors defining the triad at $B$. Phillips et al. [27] provide a summary of different methods used in the aerospace industry. In this paper, all three techniques are used to simplify the equations where necessary. In Eq. (2), $\zeta$ is the vector of four-quaternion elements used to determine the orientation of the $B$ reference frame, and $p_{B}$ is the inertial position of the $B$ reference frame. The gravitational field effects are represented by $g_{0}$.

\section{A. Summary of Governing Differential Equations}

The rigid-body formulation has three key assumptions that render invalid when dealing with very flexible vehicles:

1) Inertia properties are constant or, at best, slowly time-varying.

2) The coupling inertial force because of a rotating coordinate frame and relative velocity of flexible members is negligible. aircraft in which relative constraints are needed at the joint of the two wings).

The derivation of the EOM is based upon the principle of virtual work. The method accounts for the virtual work associated with the $B$ reference system, flexible aircraft slender (beam) structural members, and rigid bodies attached to the flexible structures. The virtual work of a beam and rigid bodies attached to a beam are initially written in terms of dependent displacement vectors. Then, the kinematic relationship between beam-dependent position vectors and the associated strains is developed. The components of virtual work are summed, and the resulting set of equations is transformed from a set of dependent position vectors and a nonminimum set of $B$ reference frame components to an independent set of strain variables and body linear and angular velocities.

The final virtual work expression, which includes both $B$ reference frame and flexible body contributions, is written

$$
\delta W=\left[\begin{array}{ll}
\delta \epsilon^{T} & \delta b^{T}
\end{array}\right]^{T}\left(-\left[\begin{array}{ll}
M_{F F} & M_{F B} \\
M_{B F} & M_{B B}
\end{array}\right]\left[\begin{array}{c}
\ddot{\epsilon} \\
\dot{\beta}
\end{array}\right]-\left[\begin{array}{ll}
C_{F F} & C_{F B} \\
C_{B F} & C_{B B}
\end{array}\right]\left[\begin{array}{c}
\dot{\epsilon} \\
\beta
\end{array}\right]+-\left[\begin{array}{ll}
K_{F F} & K_{F B} \\
K_{B F} & K_{B B}
\end{array}\right]\left[\begin{array}{c}
\epsilon \\
b
\end{array}\right]+R\right)
$$

3) External forces and moments $F_{\text {ext }}$ and $M_{\text {ext }}$, which come from aerodynamic loading, are based upon a fixed aircraft geometry. In the rigid-body case, Eq. (2) presents only inertial and external forces and moments.

For the flexible aircraft, a set of elastic equations of motion (EOM) is also introduced, which, in the context of this study, results in

$$
\begin{gathered}
M \ddot{q}+C \dot{q}+K q=R(q, \dot{q}, \lambda) \\
q=\left\{\begin{array}{c}
\epsilon \\
p_{B} \\
\Theta_{B}
\end{array}\right\} \quad \dot{q}=\left\{\begin{array}{c}
\dot{\epsilon} \\
v_{B} \\
\omega_{B}
\end{array}\right\} \quad \ddot{q}=\left\{\begin{array}{c}
\ddot{\epsilon} \\
\dot{v}_{B} \\
\dot{\omega}_{B}
\end{array}\right\}
\end{gathered}
$$

where $M$ represents generalized mass properties; $q$ is a set of generalized coordinates containing both strain $\epsilon$, associated with the flexible vehicle and the inertial position $p_{B}$, and an arbitrary orientation vector $\Theta_{B}$ of the $B$ reference frame. The matrix $C$ contains both structural damping and nonlinear terms associated with relative position and velocity terms associated with a rotating coordinate frame $\left(\omega_{B} \times v_{B}\right.$, etc. $), K$ is the stiffness matrix, and $R(q, \dot{q}, \lambda)$ represents generalized forces (including aerodynamic forces), which are a function of the finite state inflow $[28,29]$ velocities, $\lambda$. Coupling of the rigid-body and flexible dynamics occurs through the dependency of $M, C$, and $R$. Typically, the $B$ reference frame linear and angular velocities are represented by

$$
\beta=\left\{\begin{array}{c}
v_{B} \\
\omega_{B}
\end{array}\right\}
$$

The present work uses a constant strain-based formulation [30,31], which allows for airframe nonlinear geometric deformation and accounts for geometry-dependent inertia properties of the aircraft.

To develop the nonlinear governing differential equations for slender elastic structures, a systematic approach is used in which the rigid-body and elastic EOM are developed about the $B$ reference frame. The differential equations for the orientation and displacement of the $B$ reference frame are appended based upon a four-state quaternion representation. Unsteady aerodynamic modeling is included and, if required, algebraic equations for absolute or relative constraints are appended (an example of which is a joined wing where the generalized mass and damping matrices are given by

$$
\begin{gathered}
M_{F F}=J_{h \epsilon}^{T} M_{G} J_{h \epsilon} \quad M_{F B}=J_{h \epsilon}^{T} M_{G} J_{h b} \quad M_{B F}=J_{h b}^{T} M_{G} J_{h \epsilon} \\
M_{B B}=J_{h b}^{T} M_{G} J_{h b}+M_{B} \quad C_{F F}=J_{h \epsilon}^{T} M_{G} \dot{J}_{h \epsilon}+C_{G} \\
C_{F B}=J_{h \epsilon}^{T} M_{G} H_{h b}+2 J_{h \epsilon}^{T} M_{G} H_{h \dot{\epsilon} \dot{\beta}} \quad C_{B F}=J_{h b}^{T} M_{G} \dot{J}_{h \epsilon} \\
C_{B B}=J_{h b}^{T} M_{G} H_{h b}+2 J_{h b}^{T} M_{G} H_{h \dot{\epsilon} \dot{\beta}}+C_{B} \quad K_{F F}=K_{G} \\
K_{F B}=0 \quad K_{B F}=0 \quad K_{B B}=0
\end{gathered}
$$

and $M_{G}, C_{G}$, and $K_{G}$ are the assembled flexible-element generalized mass, damping, and stiffness matrices, respectively. The matrices $M_{B}$ and $C_{B}$ represent the mass and damping matrices associated with the $B$ frame rigid-element portion as described in $[32,33]$. The Jacobian matrices $J_{h \epsilon}$ and $J_{h b}$ provide relationships between flexible position and orientation vectors and the independent coordinates of strain $\epsilon$ and $B$ reference frame linear and angular velocities, $\beta$. Additional matrices $H_{h b}$ and $H_{h \dot{\epsilon} \dot{\beta}}$ capture the dynamics of a rotating coordinate frame. Complete details of the derivation are provided in $[32,33]$. Note that the traditional aircraft rigid-body EOM can be recovered from the flexible EOM ( 6 ) by assuming the elastic DOF are constant. The resultant force vector $R$ is

$$
\begin{aligned}
R & =\left\{\begin{array}{l}
R_{F} \\
R_{B}
\end{array}\right\}=\left[\begin{array}{l}
K_{F F} \\
K_{B F}
\end{array}\right] \epsilon_{\text {initial }}+\left[\begin{array}{l}
B_{g F} \\
B_{g B}
\end{array}\right] g^{B}+\left[\begin{array}{l}
B_{\mathrm{dst}_{F}}^{F} \\
B_{\mathrm{dst}_{B}}^{F}
\end{array}\right] F^{\mathrm{dst}} \\
& +\left[\begin{array}{l}
B_{\mathrm{dst}_{F}}^{M} \\
B_{\mathrm{dst}_{B}}^{M}
\end{array}\right] M^{\mathrm{dst}}+\left[\begin{array}{l}
B_{p_{F}}^{F} \\
B_{p_{B}}^{F}
\end{array}\right] F^{\mathrm{pt}}+\left[\begin{array}{l}
B_{p_{F}}^{M} \\
B_{p_{B}}^{M}
\end{array}\right] M^{\mathrm{pt}}
\end{aligned}
$$

where $\boldsymbol{\epsilon}_{\text {initial }}$ is an initial strain vector, $g^{B}$ is the body-frame $B$ resolved gravity vector, and $F^{\mathrm{dst}}, M^{\mathrm{dst}}, F^{\mathrm{pt}}$, and $M^{\mathrm{pt}}$ are bodyresolved distributed and point forces and moments. The aerodynamic forces and moments, $F^{\text {aero }}$ and $M^{\text {aero }}$, which are functions of control surface inputs, $u$, are included in $F^{\mathrm{dst}}$ and $M^{\mathrm{dst}}$. The influence matrices are derived as [31]

$$
\begin{array}{cl}
B_{\mathrm{dst}_{F}}^{F}=J_{p \epsilon}^{T} B_{F} & B_{\mathrm{dst}_{B}}^{F}=J_{p \beta}^{T} B_{F} \\
B_{\mathrm{dst}_{F}}^{M}=J_{\theta \epsilon}^{T} B_{M} & B_{\mathrm{dst}_{B}}^{M}=J_{\theta \beta}^{T} B_{M} \\
B_{p_{F}}^{F}=J_{p \epsilon}^{T} & B_{p_{B}}^{F}=J_{p \beta}^{T}
\end{array}
$$




$$
B_{p_{F}}^{M}=J_{\theta \epsilon}^{T} \quad B_{p_{B}}^{M}=J_{\theta \beta}^{T}
$$

where the matrices $B_{F}$ and $B_{M}$ are constant matrices defined by an elastic element's undeformed length. For more details, please see [31]. For simplicity, the initial strain $\epsilon_{\text {initial }}$ and point moments $M^{\mathrm{pt}}$ will be assumed to be zero. The finite strain formulation $[28,29]$ for aerodynamic forces and moments is linear in the discrete trailing edge surface deflections. These assumptions and formulations, combined with a point force representing a simplistic engine model, allow the generalized force to be expressed as [30]

$$
\begin{aligned}
R & =\left[B_{g}\right] g^{B}+\left[B_{\mathrm{dst}}^{F}\right] F^{\text {aero }}+\left[B_{\mathrm{dst}}^{M}\right] M^{\text {aero }} \\
& +\left[B_{\mathrm{dst}}^{F}\right] \frac{\partial F^{\text {aero }}}{\partial u_{\text {flap }}} u_{\text {flap }}+\left[B_{p}^{F}\right] u_{\text {thrust }}
\end{aligned}
$$

From the principle of virtual work, Eq. (ㅁ) yields

$$
\begin{gathered}
{\left[\begin{array}{ll}
M_{F F} & M_{F B} \\
M_{B F} & M_{B B}
\end{array}\right]\left[\begin{array}{c}
\ddot{\epsilon} \\
\dot{\beta}
\end{array}\right]+\left[\begin{array}{ll}
C_{F F} & C_{F B} \\
C_{B F} & C_{B B}
\end{array}\right]\left[\begin{array}{c}
\dot{\epsilon} \\
\beta
\end{array}\right]} \\
+\left[\begin{array}{ll}
K_{F F} & K_{F B} \\
K_{B F} & K_{B B}
\end{array}\right]\left[\begin{array}{l}
\epsilon \\
b
\end{array}\right]=\left\{\begin{array}{l}
R_{F} \\
R_{B}
\end{array}\right\}
\end{gathered}
$$

This set of equations contains the ones given in a compact form by Eq. (3). Note that $M=M(\epsilon), C=C(\epsilon, \dot{\epsilon}, \beta)$, and $K$ is the generalized stiffness. All the other nonlinearities are contained in the generalized force $R$. When the EOM (13) are augmented with the $B$ reference frame orientation, position, and unsteady aerodynamics, the complete set of governing differential equations is

$$
\begin{gathered}
M_{F F} \ddot{\epsilon}=-M_{F B} \dot{\beta}-C_{F F} \dot{\epsilon}-C_{F B} \beta-K_{F F} \epsilon+R_{F} \\
M_{B B} \dot{\beta}=-M_{B F} \ddot{\epsilon}-C_{B B} \beta-C_{B F} \dot{\epsilon}+R_{B} \quad \dot{\zeta}=-\frac{1}{2} \Omega_{\zeta} \zeta \\
\dot{p}_{B}=\left[\begin{array}{ll}
C^{B G} & 0
\end{array}\right] \beta \quad \dot{\lambda}=F_{1} \ddot{q}+F_{2} \dot{q}+F_{3} \lambda
\end{gathered}
$$

where $\zeta$ is a vector of four quaternion parameters used for the orientation of the $B$ reference frame, $\dot{p}_{B}$ is the inertial position vector of the $B$ reference frame (Fig. 1), $C^{B G}$ is a transformation matrix between a $B$ reference frame vector and an inertial $G$ vector, and $\lambda$ is a set of unsteady aerodynamic inflow velocities with associated differential equation matrices $F_{1}$ through $F_{3}$. For complete details, please see $[\underline{32}, \underline{33}]$.

\section{B. Aircraft Trim Solution}

Trimming is performed for both zero thrust and thrust required for 1- $g$-level flight based upon techniques outlined in[26,34]. A cost function is defined as

$$
J_{\text {trim }}=f^{T} \cdot f
$$

where for the zero thrust or gliding cases,

$$
f=\left\{\begin{array}{c}
\text { pitching moment about the origin of the } B \text { frame } \\
\text { lift weight }
\end{array}\right\}
$$

For the case of 1- $g$-level flight, the longitudinal $B$ reference frame linear and angular accelerations are used, such that

$$
f=\left\{\begin{array}{lll}
\dot{v}_{B_{y}} & \dot{v}_{B_{z}} & \dot{\omega}_{x}
\end{array}\right\}^{T}
$$

The cost function $J$ is then minimized over the solution space using the elevator deflection angle $\delta_{e}$, the body angle of attack $\alpha$, and thrust $\delta_{t}$. A simple numerical Newton-Raphson method is used to find the local minimum of the search variable; that is,

$$
\Delta \mathcal{S}_{k}=-\left[\frac{\partial f}{\partial \mathcal{S}}\right]_{k}^{-1} f_{k}
$$

where

$$
\mathcal{S}_{k}=\left\{\begin{array}{lll}
\delta_{e} & \alpha & \delta_{t}
\end{array}\right\}_{k}^{T}
$$

The search variable $\mathcal{S}$ is updated by

$$
\mathcal{S}_{k+1}=\mathcal{S}_{k}+\Delta \mathcal{S}
$$

and $f_{k+1}$ and

$$
\left[\frac{\partial f}{\partial \mathcal{S}}\right]_{k+1}^{-1}
$$

are recomputed using $\mathcal{S}_{k+1}$. The process continues until the cost function $J$ reduces to some prescribed tolerance. To prevent divergence of the solution, $\mathcal{S}_{k+1}$ is checked at each iteration step and kept within a prescribed set of bounds. The Jacobian

$$
J_{\text {trim }}=\frac{\partial f}{\partial \mathcal{S}}
$$

is computed numerically through finite differences.

\section{Solution of EOM}

To solve the nonlinear differential equations (14), a highfrequency dissipative time-stepping approach was implemented. A modified Newmark method was used and is described in [35]. The modified Newmark method was selected based upon its ability to accurately integrate large systems of equations, including ones with repeated eigenvalues, its relative ease of implementation with the current EOM modeling, and the derivation of both a first- and second-order method [36,37].

\section{Trajectory Control}

\section{A. Challenges}

Because of the nature of the very flexible aircraft construction, the wings will typically have a lower stiffness than the fuselage, generating stronger coupling of the rigid-body and structural motions in the lateral axis than in the longitudinal one. This creates a variety of challenges to be overcome by controllers. The first is the requirement of an integrated controller that handles flexibility as well as rigid-body motion. Typically, the first wing-bending mode of this class of aircraft is less than $10 \mathrm{rad} / \mathrm{s}$, creating direct interaction with classic lateral and longitudinal rigid-body aircraft modes (spiral, roll, dutch roll, and phugoid). The second major challenge is a time delay between control inputs and $B$ reference frame movement because of the flexibility of the aircraft. This time delay creates a nonminimum phase (NMP) system when the governing differential equations are linearized. The third major challenge is the introduction of additional NMP behavior because of adverse yaw from aileron inputs. The adverse yaw problem may be avoided through the use of spoilers for roll control, but that is not addressed in this work. The fourth challenge is the location of the linearized structural eigenvalues near the imaginary axis. And finally, the generalized mass and damping matrices are state-dependent.

\section{B. Requirements and Assumptions}

Before designing any controller, a set of performance objectives should be established. Currently, despite operational requirements [4], there are no published performance specification requirements for very flexible aircraft either from the military, civilian, government, or industry authorities. Given this void, it is worthwhile to refer back to the piloted aircraft military standards, MIL-STD1797A [38]. Although very flexible aircraft do not have the safety requirements inherent in piloted aircraft, minimal control performance requirements are necessary for both manned and unmanned aircraft to complete the various missions and potentially fly in the National Airspace System. For classification purposes, a very flexible vehicle will be considered a large land-based transport type aircraft, class III-L, as in Table 1 . From MIL-STD-1797A, Table 2 summarizes the desired roll requirements for level-2 flying qualities (Table $\underline{3}$ ) during takeoff, climb, loiter, and landing flight conditions. 
Table 1 Aircraft classes as defined in MIL-STD-1797A

\begin{tabular}{lll}
\hline \hline Class & \multicolumn{1}{c}{ Type of aircraft } & \multicolumn{1}{c}{ Example } \\
\hline I & $\begin{array}{c}\text { Small light aircraft } \\
\text { Medium weight, low-to- } \\
\text { II }\end{array}$ & $\begin{array}{l}\text { Primary trainer, light utility } \\
\text { Search and rescue, ISR }\end{array}$ \\
III & $\begin{array}{c}\text { Large, heavy, low-to-medium } \\
\text { maneuverability }\end{array}$ & Heavy transport, ISR \\
IV & High-maneuverability aircraft & Fighter, attack \\
\hline \hline
\end{tabular}

Table 2 Final roll performance objectives for very flexible aircraft

\begin{tabular}{lcc}
\hline \hline Flight phase & $\begin{array}{c}\text { Bank angle change/ } \\
\text { seconds to achieve }\end{array}$ & $\begin{array}{c}\text { Roll mode time } \\
\text { constant }\end{array}$ \\
\hline Climb and loiter $\mathrm{B}^{\mathrm{a}}$ & $30 \mathrm{deg} / 3.9 \mathrm{~s}$ & $3.0 \mathrm{~s}$ \\
Takeoff and landing C & $30 \mathrm{deg} / 4.0 \mathrm{~s}$ & $3.0 \mathrm{~s}$ \\
\hline \hline
\end{tabular}

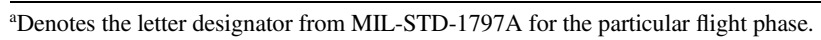

Table 3 Qualitative degrees of suitability and levels as defined in MILSTD-1797A

\begin{tabular}{ll}
\hline \hline \multicolumn{1}{c}{ Level } & \multicolumn{1}{c}{ Description } \\
\hline 1) Satisfactory & $\begin{array}{c}\text { Flying qualities clearly adequate for the mission flight } \\
\text { phase }\end{array}$ \\
2) Acceptable & $\begin{array}{c}\text { Flying qualities adequate to accomplish the mission } \\
\text { flight phase }\end{array}$ \\
3) Controllable & $\begin{array}{c}\text { Flying qualities such that the aircraft can be controlled } \\
\text { in the context of the mission flight phase; workload } \\
\text { is excessive or mission effectiveness is inadequate }\end{array}$ \\
\hline \hline
\end{tabular}

Furthermore, the maximum bank angle required shall be determined by the bank angle required to make a standard 2-min turn (turn rate of $3 \mathrm{deg} / \mathrm{s}$ or $0.05236 \mathrm{rad} / \mathrm{s}$ ). Because MIL-STD-1797A does not provide specific guidance for climb rates, a maximum climb rate of $2000 \mathrm{ft} / \min (10.16 \mathrm{~m} / \mathrm{s})$ at sea level at maximum gross weight shall be used. This rate is based upon reasonable climb rates of aircraft similar in size and weight.

Three basic maneuvers will be used to study controller performance. The first maneuver is a wings-level altitude change. The second maneuver is a steady level turn starting from a zero bank angle. And the third maneuver is a climbing turn.

Finally, the assumption will be made that the aircraft will be flying well below any divergence, flutter, or limit cycle oscillation boundaries. Whereas this assumption is restrictive for an aircraft dominated by aeroelastic effects, it is an important first step in the development of controllers for performing basic aircraft maneuvering. Future research should focus on extending the controller(s) beyond aeroelastic boundaries.

\section{Control Architecture}

As discussed in Sec. III.A, very flexible aircraft, and particularly HALE ones, present unique challenges to the design of a controller. This section details the specifics of a proposed very flexible aircraft controller.

\section{Traditional Controller Design Difficulty}

Traditional methods of modern aircraft control $[6,26,39]$ have relied upon the state vector

$x$

$$
=\left[\begin{array}{llllllllllll}
v_{B_{x}} & v_{B_{y}} & v_{B_{z}} & \omega_{B_{x}} & \omega_{B_{y}} & \omega_{B_{z}} & \Phi & \Theta & \Psi & p_{B_{x}} & p_{B_{y}} & p_{B_{z}}
\end{array}\right]^{T}
$$

or variations of $x$, in which the $B$ reference frame linear and angular velocities are $v_{B}$ and $\omega_{B}$, the classic Euler angles of roll (positive right wing down), pitch (positive nose up), and yaw (positive nose right) are given by $\Phi, \Theta$, and $\Psi$, respectively, and the inertial position is given by $p_{B}$. Linearizing the rigid body governing differential equations about this state vector and determining a constant gain matrix $K$ has been shown in numerous papers and books to yield satisfactory results when applied to nonlinear flight dynamic models. However, the current problem with additional aeroelastic effects has rendered this to be ineffective for trajectory control. In the process of developing a stabilizing controller architecture for very flexible aircraft, traditional controller designs were initially applied to a statically deformed rigid aircraft model. This controller architecture was shown to have difficulties in providing closed-loop stable trajectory tracking [33].

\section{Heuristic Approach Mimicking a Human Pilot}

Because of the difficulty in finding a stable controller for the rigid body using traditional techniques, the method was not attempted with additional elastic states. A method of decoupling the linear and nonlinear effects of the aircraft response was designed based loosely upon aircraft pilot training. A well-trained human pilot has been taught to command flight path angle $\gamma$, bank angle $\mu$, and their rates $\dot{\gamma}$ and $\dot{\mu}$ when changing altitude or heading $\Psi$, through a fast-loop (inner) and slow-loop (outer) process. The fast loop consists of commanding pitch rate, pitch angle, roll rate, and sideslip angle. These changes are commanded by the pilot, typically through the use of four controls: elevator $\delta_{e}$, aileron $\delta_{a}$, rudder $\delta_{r}$, and throttle $\delta_{t}$. Once an angle and its rate have been satisfactorily set, the pilot then performs a slow-loop (outer-loop) function of cross checking altitude, rate of climb, and other states. Heffley et al. [40] have described this type of inner- and outer-loop control as an implicit inner-loop and explicit outer-loop tracking task. Additionally, for level turns, altitude changes, and landing flair maneuvers, Heffley and Schulman [41] have provided pilot models for outer-loop control.

In similar fashion, this work proposes building a stable inner loop, using both linear and nonlinear theory, in which the states are the linear and angular $B$ reference frame velocities $\beta$ [Eq. (5)], and augmented error states. The outer loop or slow loop is then controlled through a nonlinear transformation and traditional proportional, integral, derivative (PID) control techniques [42]. Conceptually, the entire system is shown in Fig. 2. In the figure, $\beta_{0}$ are the initial trimmed $B$ reference frame linear and angular velocities. For the outer loop, a commanded $B$ reference frame velocity, $\beta_{\text {com }}$, and differences are generated

$$
\Delta \beta_{\text {com }}=\beta_{\text {com }}-\beta
$$

and used to excite inner-loop integrator error states. Also, as indicated in Fig. 2, the subscripts des and com are the desired and commanded values, respectively. For the inner loop, the difference in $\beta$

$$
\Delta \beta=\beta-\beta_{0}
$$

is required to generate differences in the control vector from the initial control deflections $u_{0}$, such that

$$
u=u_{0}+\Delta u
$$

and $\Delta u$ is a function of the gain matrix $K$ and the augmented error states $e$ :

$$
\Delta u=K(\Delta \beta, e)
$$

\section{Nonlinear Transformation from Flight Path and Bank Angles to Body Velocities}

The classic rigid-body aircraft kinematic equations used for the nonlinear transformation are $[\underline{26}, \underline{39}, \underline{43}]$

$$
V=\sqrt{u^{2}+v^{2}+w^{2}}
$$




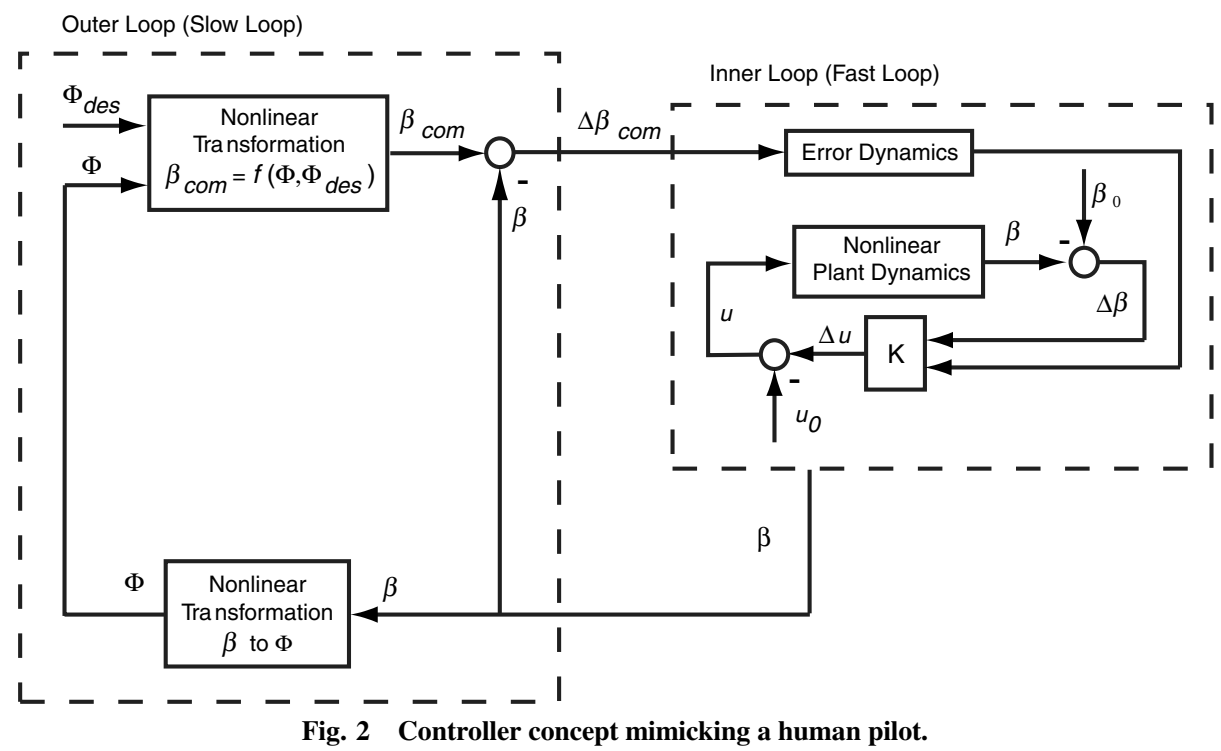

$$
\beta_{s}=\sin ^{-1}(v / V)
$$

$$
\begin{aligned}
\gamma & =\sin ^{-1}\left[(\cos \alpha \sin \Theta-\sin \alpha \cos \Theta \cos \Phi) \cos \beta_{s}\right. \\
& \left.-\cos \Theta \sin \Phi \sin \beta_{s}\right]
\end{aligned}
$$

$\mu$

$$
=\sin ^{-1}\left(\frac{\cos \Theta \sin \Phi \cos \beta_{s}+(\cos \alpha \sin \Theta-\sin \alpha \cos \Theta \cos \Phi) \sin \beta_{s}}{\cos \gamma}\right)
$$

$$
\begin{gathered}
\dot{\Phi}=P+\tan \Theta(Q \sin \Phi+R \cos \Phi) \\
\dot{\Theta}=Q \cos \Phi-R \sin \Phi
\end{gathered}
$$

where $V$ is the total airspeed, and the classic aircraft longitudinal, lateral, and vertical velocity components are $u$ positive out the nose, $v$ positive out the right wing, and $w$ positive down. Further, $\gamma$ is the flight path elevation angle, $\alpha$ is the aircraft angle of attack, $\mu$ is the flight path bank angle, and $\beta_{s}$ is the sideslip angle. The classic aircraft angular rates are roll rate $P$, pitch rate $Q$, and yaw rate $R$. The dynamic equations of interest are

$$
\begin{gathered}
L=\frac{1}{2} \rho V^{2} S C_{L_{\alpha}} \alpha \\
\dot{v}=-R u+P w+g_{0} \sin \Phi \cos \Theta+\frac{F_{y}}{m}
\end{gathered}
$$

where $L$ is the aircraft lift, $\rho$ is the atmospheric density, $S$ is the surface area of the wing, $C_{L_{\alpha}}$ is the equivalent aircraft lift curve slope, $g_{0}$ is the magnitude of the gravity vector, $F_{y}$ is the lateral force because of aerodynamic and control inputs, and $m$ is the aircraft mass. Equation (33) is a steady-state lift approximation and will be used to develop an angle-of-attack $\alpha$ dynamic relationship. Equation (34) is the rigid-body lateral acceleration EOM and will be used to develop relationships for the $B$ reference frame angular velocities. The angle of attack and sideslip are related to the longitudinal velocities as

$$
\left\{\begin{array}{c}
u \\
v \\
w
\end{array}\right\}=\left\{\begin{array}{c}
V \cos (\alpha) \cos \left(\beta_{s}\right) \\
V \sin \left(\beta_{s}\right) \\
V \sin (\alpha) \cos \left(\beta_{s}\right)
\end{array}\right\}
$$

such that the angle of attack may be written as

$$
\alpha=\tan ^{-1}(w / u)
$$

Before Eqs. (27-36) can be used in the current formulation, transformations of the linear and angular body velocities and quaternion to classic Euler angles are presented. For the linear and angular velocities, the relationships are

$$
\left\{\begin{array}{l}
v_{B_{x}} \\
v_{B_{y}} \\
v_{B_{z}}
\end{array}\right\}=\left[\begin{array}{ccc}
0 & 1 & 0 \\
1 & 0 & 0 \\
0 & 0 & -1
\end{array}\right]\left\{\begin{array}{c}
u \\
v \\
w
\end{array}\right\}
$$

and

$$
\left\{\begin{array}{l}
\omega_{B_{x}} \\
\omega_{B_{y}} \\
\omega_{B_{z}}
\end{array}\right\}=\left[\begin{array}{ccc}
0 & 1 & 0 \\
1 & 0 & 0 \\
0 & 0 & -1
\end{array}\right]\left\{\begin{array}{l}
P \\
Q \\
R
\end{array}\right\}
$$

To develop the quaternion relationship, first the body $B$ to inertial $G$ rotation matrix in terms of the Euler angles is found as

$$
\begin{aligned}
& C^{G B} \\
& \quad=\left[\begin{array}{ccc}
(c \Phi c \Psi+s \Phi s \Theta s \Psi) & (-c \Phi s \Psi+s \Phi s \Theta c \Psi) & -s \Phi c \Theta \\
c \Theta s \Psi & c \Theta c \Psi & s \Theta \\
(s \Phi c \Psi-c \Phi s \Theta s \Psi) & -(s \Phi s \Psi+c \Phi s \Theta c \Psi) & c \Phi c \Theta
\end{array}\right]
\end{aligned}
$$

where the shorthand $\cos \Phi \equiv c \Phi$ and $\sin \Phi \equiv s \Phi$ is used. The Euler angles of interest have the following relationship to the quaternions [33]:

$$
\begin{gathered}
\sin \Theta=2\left(\zeta_{2} \zeta_{3}+\zeta_{0} \zeta_{1}\right) \\
\cos \Theta \cos \Phi=\zeta_{0}^{2}-\zeta_{1}^{2}-\zeta_{2}^{2}+\zeta_{3}^{2} \\
\cos \Theta \sin \Phi=2\left(-\zeta_{1} \zeta_{3}+\zeta_{0} \zeta_{2}\right)
\end{gathered}
$$

To perform the transformation from given inputs of flight path angle and bank angle to desired body velocities, the following assumptions are made:

1) The angle of sideslip $\beta_{s}$ is zero.

2) The Euler bank angle $\Phi$ will be used in place of bank angle $\mu$.

3 ) Total velocity $V$ is prescribed and typically assumed constant.

4) The angle of attack $\alpha$ is proportional to $\cos \Phi$. 
5) Lateral side forces are only a function of cross coupling of linear and angular velocities and gravity component because of Euler bank and pitch angle, and lateral velocity is constant.

Whereas zero sideslip, assumption 1, is typically a requirement of manned aircraft for comfort reasons, it is also desired to minimize coupling of the lateral and longitudinal aircraft motion. Assumption 2 is justified by applying small angle assumptions to $\gamma$, Euler pitch angle $\Theta$, and angle of attack $\alpha$. Using these assumptions and Eq. (30),

$$
\mu \approx \Phi
$$

Further, if a navigational loop is wrapped around the architecture described in Fig. 2, the Euler roll angle would be convenient as an input. Assumption 3 is made to simplify the resulting nonlinear transformation equations. Assumption 4 has two significant effects. The first is that unsteady aerodynamic effects presented in [32] are neglected. This is done because one of the goals of this work is to develop an initial control architecture for very flexible aircraft, without consideration of aeroelastic boundaries. Additionally, the controller is designed to maintain aircraft velocities well below these aeroelastic boundaries, preventing destabilization of the controller through unmodeled dynamics (within the controller). The second significant effect of assumption 4 is that an increase in angle of attack to generate additional lift for a wings-level climb is not considered. This is reasonable approximation used in most rigid-body aircraft performance equations [43], where

$$
\text { rate of climb }=\frac{\text { excess thrust }}{\text { weight }}
$$

Assumption 4 is derived from the steady level-turn performance equation [43]:

$$
n_{\text {load }}=\frac{1}{\cos \Phi}
$$

where the load factor $n_{\text {load }}$ is the nondimensionalized acceleration of gravity. Assuming linear three-dimensional lift theory and a level turn,

$$
L_{\Phi}=n_{\text {load }} L_{\Phi}=0
$$

Using Eq. (33) in Eq. (46), it can be shown that

$$
\alpha_{\Phi}=\frac{1}{\cos \Phi} \alpha_{0}
$$

where $\alpha_{0}$ is the angle of attack corresponding to a wings level at steady level flight. Finally, assumption 5, which simplifies the lateral EOM (34), provides one of three resulting equations used to determine the $B$ reference frame angular velocities.

Using Eqs. (27-47) and the five assumptions, the resulting set of equations are as follows:

1) Angle-of-attack relationships:

$$
\alpha=\frac{1}{\cos \Phi} \alpha_{0} \quad \dot{\alpha}=\tan \Phi \sec \Phi \alpha_{0} \dot{\Phi}
$$

2) Linear body velocity relationships:

$$
\begin{array}{ll}
v_{B_{z}}=-V \sin \alpha & \dot{v}_{B_{z}}=-V \cos \alpha \dot{\alpha} \\
v_{B_{y}}=\sqrt{V^{2}-v_{B_{z}}^{2}} & \dot{v}_{B_{y}}=-\frac{v_{B_{z}} \dot{v}_{B_{z}}}{v_{B_{y}}}
\end{array}
$$

3) Flight path angle, Euler pitch angle, and angle-of-attack relationships:

$$
\sin \gamma=\cos \alpha \sin \Theta-\cos \Phi \sin \alpha \cos \Theta
$$

$$
\begin{aligned}
\dot{\Theta} & =\frac{\cos \gamma \dot{\gamma}+(\sin \alpha \sin \Theta+\cos \Phi \cos \alpha \cos \Theta) \dot{\alpha}}{\cos \alpha \cos \Theta+\cos \Phi \sin \alpha \sin \Theta} \\
& -\frac{\sin \Phi \sin \alpha \cos \Theta \dot{\Phi}}{\cos \alpha \cos \Theta+\cos \Phi \sin \alpha \sin \Theta}
\end{aligned}
$$

4) Body angular velocity relationships:

$$
\begin{gathered}
\dot{\Phi}=(\tan \Theta \sin \Phi) \omega_{B_{x}}+\omega_{B_{y}}-(\tan \Theta \cos \Phi) \omega_{B_{z}} \\
g_{0} \sin \Phi \cos \Theta=v_{B_{z}} \omega_{B_{y}}-v_{B_{y}} \omega_{B_{z}} \\
\dot{\Theta}=\cos \Phi \omega_{B_{x}}+\sin \Phi \omega_{B_{z}}
\end{gathered}
$$

Given the flight path angle and rate $\gamma$ and $\dot{\gamma}$ and the Euler roll angle and rate $\Phi$ and $\dot{\Phi}$, Eqs. (48-55) are used to solve for the commanded $B$ reference velocities, $\overline{\beta_{\text {com }}}$.

To close the outer loop of Fig. $\underline{2}$, the actual flight path angle $\gamma$, Euler bank angle $\Phi$, and their rates $\dot{\gamma}$ and $\dot{\Phi}$ are determined. Differences are then found:

$$
\begin{array}{cc}
\Delta \gamma & =\gamma-\gamma_{\mathrm{des}} \quad \Delta \dot{\gamma}=\dot{\gamma}-\dot{\gamma}_{\mathrm{des}} \\
\Delta \Phi=\Phi-\Phi_{\mathrm{des}} & \Delta \dot{\Phi}=\dot{\Phi}-\dot{\Phi}_{\mathrm{des}}
\end{array}
$$

The commanded flight path elevation angle and Euler roll angle are computed using a traditional linear technique of PID control:

$$
\begin{array}{cl}
\gamma_{\text {com }}=\gamma_{\text {des }}+\Delta \gamma_{\text {com }} & \dot{\gamma}_{\text {com }}=\dot{\gamma}_{\text {des }}+\Delta \dot{\gamma}_{\text {com }} \\
\Phi_{\text {com }}=\Phi_{\text {des }}+\Delta \Phi_{\text {com }} & \dot{\Phi}_{\text {com }}=\dot{\Phi}_{\text {des }}+\Delta \dot{\Phi}_{\text {com }}
\end{array}
$$

where

$$
\begin{aligned}
& \Delta \gamma_{\text {com }}=k_{\gamma} \Delta \gamma+k_{I \gamma} \int_{0}^{t}(\Delta \gamma) \mathrm{d} \tau+k_{I I \gamma} \int_{0}^{t}\left(\int_{0}^{t}(\Delta \gamma) \mathrm{d} \tau\right) \mathrm{d} \tau \\
& \quad+k_{d \gamma} \Delta \dot{\gamma} \quad \Delta \dot{\gamma}_{\text {com }}=k_{\dot{\gamma}} \Delta \dot{\gamma}+k_{I \dot{\gamma}} \Delta \gamma+k_{I I \dot{\gamma}} \int_{0}^{t}(\Delta \gamma) \mathrm{d} \tau \\
& \quad+k_{I I I \dot{\gamma}} \int_{0}^{t}\left(\int_{0}^{t}(\Delta \gamma) \mathrm{d} \tau\right) \mathrm{d} \tau \\
& \Delta \Phi_{\text {com }}=k_{\Phi} \Delta \Phi+k_{I \Phi} \int_{0}^{t}(\Delta \Phi) \mathrm{d} \tau+k_{d \Phi} \Delta \dot{\Phi} \\
& \Delta \dot{\Phi}_{\text {com }}=k_{\dot{\Phi}} \Delta \dot{\Phi}+k_{I \dot{\Phi}} \Delta \Phi+k_{I I \dot{\Phi}} \int_{0}^{t}(\Delta \Phi) \mathrm{d} \tau
\end{aligned}
$$

The outer-loop feedback gains, $k_{\gamma}, k_{I \gamma}$, and so on, of Eq. (60), are initially determined using the single-input/single-output heuristic guidelines of Ziegler and Nichols [44,45], where

$$
u=k_{c} e+k_{I} \int e \mathrm{~d} t+k_{d} \dot{e}
$$

For PID and PI controllers, Ziegler and Nichols's recommend values are given in Table 4 , in which $k_{u}$ is the closed-loop gain required to make the system marginally stable, and $t_{u}$ is the corresponding period between oscillations. The gains are then adjusted to meet desired performance. Because of the potential instability of integral feedback [느] , the additional integral gains $\left(k_{\mathrm{II}}, k_{\mathrm{II} \dot{\gamma}}, k_{\mathrm{III} \dot{\gamma}}\right.$, etc.) are

Table 4 Ziegler and Nichols $[\underline{44}, \underline{45}]$ PID and PI tuning parameters

\begin{tabular}{lcc}
\hline \hline & PID & PI \\
\hline Proportional gain & $k_{c}=0.6 k_{u}$ & $k_{c}=0.45 k_{u}$ \\
Integral gain & $k_{I}=k_{c} / 0.5 t_{u}$ & $k_{I}=k_{c} / 0.85 t_{u}$ \\
Derivative gain & $k_{d}=\frac{1}{8} k_{c} t_{u}$ & - \\
\hline \hline
\end{tabular}


chosen to be at least an order or more of magnitude less than the first integral gains ( $k_{\mathrm{I} \gamma}, k_{\mathrm{I} \dot{\gamma}}$, and so on).

\section{Modification of Proposed Control Architecture}

This section presents a method for extending the control architecture previously introduced to a flexible aircraft. First a separation of the lateral and longitudinal motion is presented. Then control techniques are applied to the lateral and longitudinal motion separately. Finally, coupling between the lateral and longitudinal motion is shown to be handled in an outer-loop strategy.

Section III.C outlined a control strategy for very flexible aircraft. The philosophy of this approach is to provide a systematic method in which the dominant coupling of lateral and longitudinal motion is handled in a slower outer loop, and $B$ reference frame linear and angular velocities are handled in a faster inner loop. The six states (linear and angular $B$ reference frame velocities) of the inner loop are typically easily separated into longitudinal and lateral motion with corresponding control effectors. This allows individual control schemes to be applied to each set of the inner-loop dynamics (longitudinal and lateral). The outer loop controls the required longitudinal and lateral motion necessary for trajectories when this motion is coupled (i.e., steady level turns and climbing turns). Additionally, the outer loop handles coupling of the lateral and longitudinal motion because of gravitational effects. Although this idea is not new and has been applied to high angle-of-attack flight [46-48], it typically has not been applied to low-to-moderate angleof-attack flight regimes [47]. This is due largely to traditional aircraft control design assuming relatively small to moderate amounts of nonlinear cross coupling. With very flexible aircraft, the large potential movement of the center of mass from the origin of the $B$ reference frame can create a significant nonlinear coupling.

\section{Separation of Lateral and Longitudinal Motion}

As shown by Shearer and Cesnik [32] and Shearer [33], for this class of vehicles with relatively stiff fuselages, longitudinal motion does not appear to be significantly affected by wing flexibility. So it is assumed that wing flexibility is a secondary and minimal contribution to aircraft longitudinal motion. Using this assumption and the previous assumptions of Sec. III.C, a separation between longitudinal and lateral/elastic motion is made. Additionally, an examination of the rigid-body LTI state matrices [33] supports this assumption of minimal cross coupling between the lateral $\left(v_{B_{x}}, \omega_{B_{y}}\right.$, and $\left.\omega_{B_{z}}\right)$ and the longitudinal $\left(v_{B_{y}}, v_{B_{z}}\right.$, and $\left.\omega_{B_{x}}\right)$ states. An eigenvalue analysis of a representative HALE aircraft further supports this assumption.
The lateral and the longitudinal eigenvalues and eigenvectors of the $B$ reference frame for a static and elastically deformed wing at a given (heavy) fuel weight are presented in Tables $\underline{5}$ and $\underline{6}$. As can be seen by examining the eigenvectors, there exists a fairly distinct separation of lateral and longitudinal motion.

When the elastic wing states are included, a distinct change in the lateral eigenvalues and eigenvectors occurs, as seen in Table 5. The sideslip and sideslip/yaw-rate modes experience a significant change in the eigenvalues and changes in the sign of the eigenvector components. However, the real change is in the roll mode, as it no longer has any significant contribution from the rigid-body roll rate $\omega_{B_{y}}$. Rather, $\omega_{B_{y}}$ now contributes to various elastic strains and rates (not presented). Because of this, the elastic states are assumed to be tightly coupled with the rigid-body lateral motion. The longitudinal eigenvalues and vectors also experience a change as seen in Table $\underline{6}$, with a major change in the vertical/longitudinal eigenvalue. This eigenvalue now has contributions of about $2 \%$ from several of the inand out-of-plane bending strain rates (not presented). However, from Table 6 , over $94.5 \%$ of the contribution comes from the $B$ reference frame longitudinal states. Because of this significant contribution, it assumed that the longitudinal states are decoupled from the elastic states. Although this strong coupling of lateral and elastic modes cannot be generalized for all very flexible aircraft, the analysis does hold for the representative aircraft used here at different loadings (fuel) conditions (not shown). In general, an eigenvalue and eigenvector analysis should be performed at nonlinear equilibrium conditions of a very flexible aircraft configuration to determine the coupling of elastic states with $B$ reference frame motion. Based upon the outcome of the analysis, various linear and nonlinear control schemes could be used. For example, Gregory [22-25] has developed filtered nonlinear control techniques and optimum sensor placement for longitudinal control with coupled aeroelastic effects. In this paper, an LQR formulation is developed for the lateral motion, and a nonlinear dynamic inversion is developed for the longitudinal motion.

\section{Lateral Motion Inner-Loop Controller}

Because of the inherent NMP zeros present in the lateral dynamics, most nonlinear control schemes are not sufficient for controlling it. Because of this, a traditional LQR controller is used for the inner-loop lateral motion. For the lateral controller, full state feedback was assumed, in which the states to be controlled are

$$
x_{\text {lat }}=\left[\begin{array}{lllll}
\epsilon^{T} & \dot{\epsilon}^{T} & v_{B_{x}} & \omega_{B_{y}} & \omega_{B_{z}}
\end{array}\right]^{T}
$$

Table 5 Lateral eigenvalues and eigenvectors for flexible aircraft with a rigid fuselage

\begin{tabular}{|c|c|c|c|}
\hline Type & $\begin{array}{c}\text { Eigenvalue, rad/s, statically/elastically } \\
\text { deformed wing }\end{array}$ & \multicolumn{2}{|c|}{$\begin{array}{c}\text { Normalized eigenvector statically/elastically } \\
\text { deformed wing }\end{array}$} \\
\hline Sideslip & $-2.508 \times 10^{-4} /-1.549 \times 10^{-1}$ & $\begin{array}{c}9.973 \times 10^{-1} /-9.957 \times 10^{-1} \\
-4.075 \times 10^{-12} /-6.420 \times 10^{-13} \\
5.415 \times 10^{-13} /-1.859 \times 10^{-13} \\
2.988 \times 10^{-14} /-2.315 \times 10^{-14} \\
-2.303 \times 10^{-3} / 4.711 \times 10^{-2} \\
-7.347 \times 10^{-2} /-8.022 \times 10^{-2} \\
9.900 \times 10^{-1} /-9.916 \times 10^{-1}\end{array}$ & $\begin{array}{l}v_{B_{x}} \\
v_{B_{y}} \\
v_{B_{z}} \\
\omega_{B_{x}} \\
\omega_{B_{y}} \\
\omega_{B_{z}} \\
v_{B_{x}}\end{array}$ \\
\hline Sideslip/yaw rate & $-1.013 / 5.305 \times 10^{-2}$ & $\begin{array}{c}1.978 \times 10^{-15} / 7.062 \times 10^{-12} \\
5.455 \times 10^{-15} / 1.797 \times 10^{-12} \\
1.844 \times 10^{-14} / 9.341 \times 10^{-14} \\
-5.876 \times 10^{-2} /-1.863 \times 10^{-2} \\
1.280 \times 10^{-1} / 1.277 \times 10^{-1}\end{array}$ & $\begin{array}{l}v_{B_{y}} \\
v_{B_{z}} \\
\omega_{B_{x}} \\
\omega_{B_{y}} \\
\omega_{B_{z}}\end{array}$ \\
\hline Roll rate & -6.7172 & $\begin{array}{c}2.904 \times 10^{-2} \\
-3.415 \times 10^{-13} \\
9.502 \times 10^{-12} \\
-8.748 \times 10^{-13} \\
9.996 \times 10^{-1} \\
4.806 \times 10^{-3}\end{array}$ & $\begin{array}{l}v_{B_{x}} \\
v_{B_{y}} \\
v_{B_{z}} \\
\omega_{B_{x}} \\
\omega_{B_{y}} \\
\omega_{B_{z}}\end{array}$ \\
\hline
\end{tabular}


Table 6 Longitudinal eigenvalues and eigenvectors for flexible aircraft with a rigid fuselage

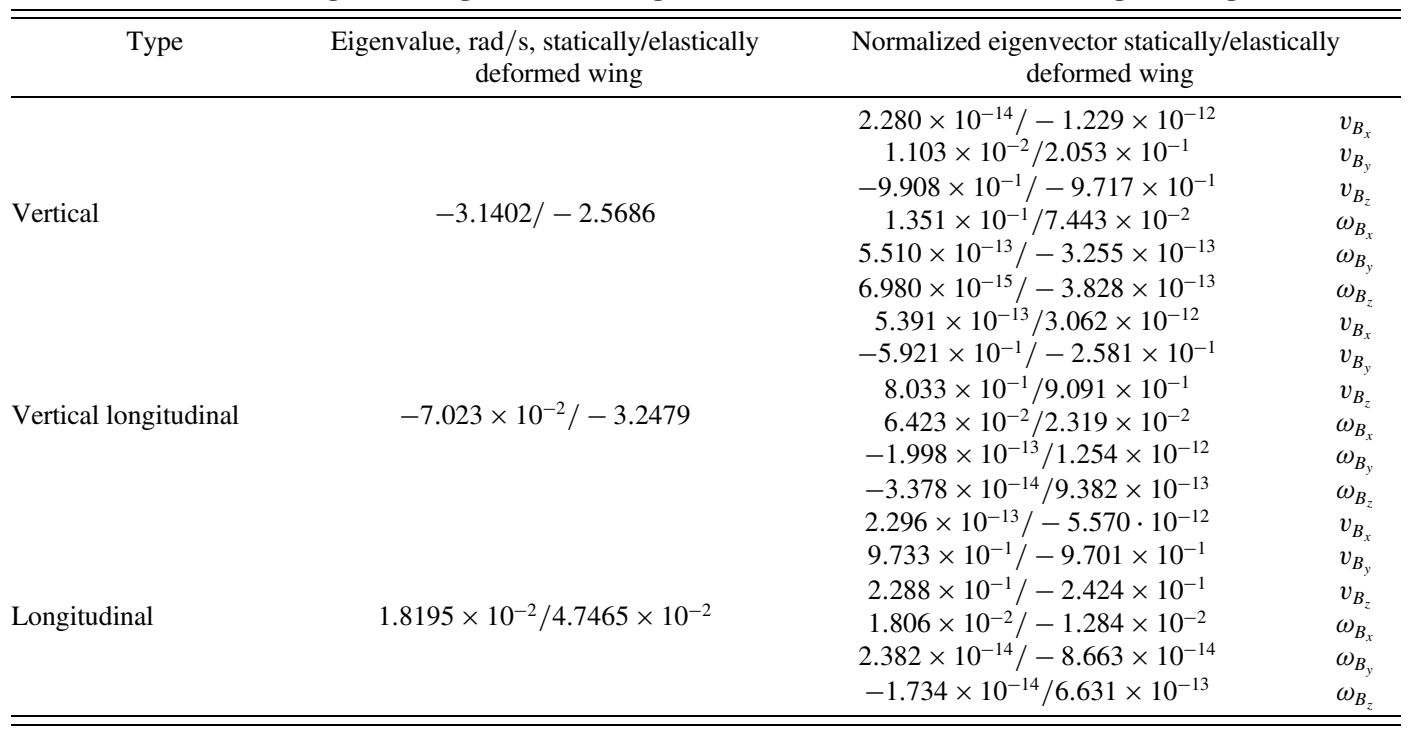

Although the assumption of full elastic state feedback ( $\epsilon$ and $\dot{\epsilon})$ may present a practical limitation, it is used here as a starting point for very flexible aircraft control architecture development. Linear time invariant $A$ and $B$ matrices are generated from Eq. (14) for the lateral states of Eq. (62). Error states are then augmented to the system such that

$$
x_{\text {lat }}=\left[\begin{array}{llllll}
\epsilon^{T} & \dot{\epsilon}^{T} & v_{B_{x}} & \omega_{B_{y}} & \omega_{B_{z}} & e_{\text {lat }}^{T}
\end{array}\right]^{T}
$$

and

$$
\dot{e}_{\text {lat }}=\left\{\begin{array}{c}
v_{B_{x}}-0 \\
\omega_{B_{y}}-\omega_{B_{y} \mathrm{com}} \\
\omega_{B_{z}}-\omega_{B_{z} \mathrm{com}}
\end{array}\right\}
$$

The augmented system can then be represented as

$$
\dot{x}_{\text {lat }}=\left[\begin{array}{cc}
A_{\text {lat }} & 0 \\
C_{\text {lat }} & 0
\end{array}\right] x_{\text {lat }}+\left[\begin{array}{c}
B_{\text {lat }} \\
0
\end{array}\right] u_{\text {lat }}+\left[\begin{array}{c}
0 \\
-I
\end{array}\right]\left\{\begin{array}{c}
0 \\
\omega_{B_{y} \text { com }} \\
\omega_{B_{z} \text { com }}
\end{array}\right\}
$$

where

$$
C_{\text {lat }}=\left[\begin{array}{ccccc}
0 & 0 & 1 & 0 & 0 \\
0 & 0 & 0 & 1 & 0 \\
0 & 0 & 0 & 0 & 1
\end{array}\right]
$$

The lateral control vector is found using the control law

$$
u_{\text {lat }}=-\left[\begin{array}{ll}
K_{\text {lat }} & K_{e_{\text {lat }}}
\end{array}\right]\left\{\begin{array}{l}
x_{\text {lat }} \\
e_{\text {lat }}
\end{array}\right\}
$$

where $K_{\text {lat }}$ and $K_{e_{\text {lat }}}$ are found using standard LQR techniques applied to the augmented system of Eq. (65).

\section{Longitudinal Motion Inner-Loop Controller}

In a similar manner to Al-Hiddabi [49] and Al-Hiddabi and McClamroch [50], partial feedback linearization or dynamic inversion is used for development of the very flexible aircraft. Here, dynamic inversion is applied to the longitudinal states of interest; that is,

$$
x_{\text {long }}=\left[\begin{array}{lll}
v_{B_{y}} & v_{B_{z}} & \omega_{B_{x}}
\end{array}\right]^{T}
$$

For the very flexible aircraft, a subset of the linear and angular body velocities, $\beta$, are desired to be controlled [Eq. (68)] using dynamic inversion. To accomplish this, $\beta$ is found from the EOM for $\epsilon$ and $\beta$. Starting with Eq. (14),

$$
\begin{aligned}
& {\left[\begin{array}{ll}
M_{F F} & M_{F B} \\
M_{B F} & M_{B B}
\end{array}\right]\left\{\begin{array}{l}
\ddot{\epsilon} \\
\dot{\beta}
\end{array}\right\}} \\
& =-\left[\begin{array}{ll}
C_{F F} & C_{F B} \\
C_{B F} & C_{B B}
\end{array}\right]\left\{\begin{array}{l}
\dot{\epsilon} \\
\beta
\end{array}\right\}-\left\{\begin{array}{c}
K_{F F} \epsilon \\
0
\end{array}\right\}+\left\{\begin{array}{l}
R_{F} \\
R_{B}
\end{array}\right\}
\end{aligned}
$$

these can be solved by inverting the generalized mass matrix. Using Fact 2.15.3 of [51],

$$
\begin{aligned}
& {\left[\begin{array}{ll}
A & B \\
B^{T} & C
\end{array}\right]^{-1}} \\
& =\left[\begin{array}{cc}
\left(A-B C^{-1} B^{T}\right)^{-1} & -\left(A-B C^{-1} B^{T}\right)^{-1} B C^{-1} \\
-C^{-1} B^{T}\left(A-B C^{-1} B^{T}\right)^{-1} & C^{-1} B^{T}\left(A-B C^{-1} B^{T}\right)^{-1} B C^{-1}+C^{-1}
\end{array}\right]
\end{aligned}
$$

and noting $M_{F B}=M_{B F}^{T}$ from Eq. (7), the inverse of the generalized mass matrix of Eq. ( $\underline{69})$ is

$$
\begin{aligned}
& {\left[\begin{array}{ll}
M_{F F} & M_{F B} \\
M_{B F} & M_{B B}
\end{array}\right]^{-1}} \\
& \quad=\left[\begin{array}{cc}
Q_{M} & -Q_{M} M_{F B} M_{B B}^{-1} \\
-M_{B B}^{-1} M_{B F} Q_{M} & M_{B B}^{-1} M_{B F} Q_{M} M_{F B} M_{B B}^{-1}+M_{B B}^{-1}
\end{array}\right]
\end{aligned}
$$

where

$$
Q_{M}=\left(M_{F F}-M_{F B} M_{B B}^{-1} M_{B F}\right)^{-1}
$$

Therefore, $\ddot{\epsilon}$ and $\dot{\beta}$ are given by

$$
\ddot{\epsilon}=-C_{11} \dot{\epsilon}-C_{12} \beta-Q_{M} K_{F F} \epsilon+R_{F 1}
$$

$$
\dot{\beta}=-C_{22} \beta-C_{21} \dot{\epsilon}+R_{B 1}
$$

where 


$$
\begin{gathered}
C_{11}=Q_{M} C_{F F}-Q_{M} M_{F B} M_{B B}^{-1} C_{B F} \\
C_{12}=Q_{M} C_{F B}-Q_{M} M_{F B} M_{B B}^{-1} C_{B B} \\
C_{21}=-M_{B B}^{-1} M_{B F} Q_{M} C_{F F}+\left(M_{B B}^{-1} M_{B F} Q_{M} M_{F B} M_{B B}^{-1}+M_{B B}^{-1}\right) C_{B F} \\
C_{22}=-M_{B B}^{-1} M_{B F} Q_{M} C_{F B}+\left(M_{B B}^{-1} M_{B F} Q_{M} M_{F B} M_{B B}^{-1}+M_{B B}^{-1}\right) C_{B B} \\
R_{F 1}=Q_{M} R_{F}-Q_{M} M_{F B} M_{B B}^{-1} R_{B} \\
R_{B 1}=-M_{B B}^{-1} M_{B F} Q_{M} R_{F}+\left(M_{B B}^{-1} M_{B F} Q_{M} M_{F B} M_{B B}^{-1}+M_{B B}^{-1}\right) R_{B}
\end{gathered}
$$

The generalized force vectors $R_{F}$ and $R_{B}$ can be further expanded into a control affine form:

$$
\begin{aligned}
& R_{F}=R_{F_{u=0}}(\epsilon, \dot{\epsilon}, \beta, \zeta, \lambda)+R_{F_{u}}(\epsilon, \dot{\epsilon}, \beta, \zeta, \lambda) u \\
& R_{B}=R_{B_{u}=0}(\epsilon, \dot{\epsilon}, \beta, \zeta, \lambda)+R_{B_{u}}(\epsilon, \dot{\epsilon}, \beta, \zeta, \lambda) u
\end{aligned}
$$

The body velocity equation (74) can then be written in the generalized form:

$$
\dot{\beta}=f_{\beta}+g_{\beta} u
$$

where

$$
\begin{aligned}
f_{\beta} & =-C_{22} \beta-C_{21} \dot{\epsilon}-M_{B B}^{-1} M_{B F} Q_{M} R_{F_{u=0}} \\
& +\left(M_{B B}^{-1} M_{B F} Q_{M} M_{F B} M_{B B}^{-1}+M_{B B}^{-1}\right) R_{B_{u=0}}
\end{aligned}
$$

and

$$
g_{\beta}=-M_{B B}^{-1} M_{B F} Q_{M} R_{F_{u}}+\left(M_{B B}^{-1} M_{B F} Q_{M} M_{F B} M_{B B}^{-1}+M_{B B}^{-1}\right) R_{B_{u}}
$$

Stability of dynamic inversion requires the so-called internal or zero dynamics [52] to be stable. For the partial dynamic inversion scheme proposed, the internal dynamics consist of the controlled lateral states and the uncontrolled inflow states [32]. The assumption is made that the internal dynamic states are stable. This assumption is valid provided the linear controller of Sec. III.D. 2 yields closed-loop stability, and the inflow states $\lambda$ are not driven unstable because of the aeroelastic boundaries of flutter and divergence. Although avoiding the aeroelastic boundaries is a limitation of the current design, it is a necessary requirement in the developing stages of controller design for very flexible aircraft.

The longitudinal outputs to be tracked [Eq. (68)] can be written as

$$
y_{\text {long }}=C_{\text {long }} \beta
$$

where

$$
C_{\text {long }}=\left[\begin{array}{llllll}
0 & 1 & 0 & 0 & 0 & 0 \\
0 & 0 & 1 & 0 & 0 & 0 \\
0 & 0 & 0 & 1 & 0 & 0
\end{array}\right]
$$

The linearized form is

$$
\dot{\chi}_{\text {long }}=v_{\text {long }}
$$

where

$$
u_{\text {long }}=g_{\beta}^{-1}\left(v_{\text {long }}-f_{\beta}\right)
$$

The system is then augmented with longitudinal error states

$$
\dot{e}_{\text {long }}=\left\{\begin{array}{c}
v_{B_{y}}-v_{B_{y} \mathrm{com}} \\
v_{B_{z}}-v_{B_{z} \mathrm{com}} \\
\omega_{B_{x}}-\omega_{B_{x} \mathrm{com}}
\end{array}\right\}
$$

and the final linearized system is

$$
\begin{gathered}
\left\{\begin{array}{c}
\dot{\chi}_{\text {long }} \\
\dot{e}_{\text {long }}
\end{array}\right\}=\left[\begin{array}{cc}
0 & 0 \\
C_{\text {long }} & 0
\end{array}\right]\left\{\begin{array}{l}
\chi_{\text {long }} \\
e_{\text {long }}
\end{array}\right\}+\left[\begin{array}{c}
I \\
0
\end{array}\right]\left\{v_{\text {long }}\right\} \\
+\left[\begin{array}{c}
0 \\
-I
\end{array}\right]\left\{\begin{array}{c}
v_{B_{y} \text { com }} \\
v_{B_{z} \text { com }} \\
\omega_{B_{x} \text { com }}
\end{array}\right\}
\end{gathered}
$$

The control variable $v_{\text {long }}$ is then computed using full state feedback as

$$
v_{\text {long }}=-\left[\begin{array}{ll}
K_{\chi_{\text {long }}} & K_{e_{\text {long }}}
\end{array}\right]\left\{\begin{array}{l}
\chi_{\text {long }} \\
e_{\text {long }}
\end{array}\right\}
$$

where the gains $K_{\chi_{\text {long }}}$ and $K_{e_{\text {long }}}$ are computed using the LQR technique. The actual control signal $u_{\text {long }}$ is computed using Eq. (84).

Finally, because dynamic inversion becomes unstable with the introduction of NMP zeros [53], flight path angle is tracked, instead of altitude, in the outer-loop design to prevent the introduction of NMP zeros into the inner-loop longitudinal dynamics. This is necessary because in traditional wing/body/tail aircraft, an increase in altitude is accomplished by deflecting the trailing edge surface of the elevator upwards. This creates a downward force which temporarily decreases the overall aircraft lift and a subsequent decrease in altitude. However, this downward force also creates a nose up pitching moment, which increases the angle of attack. Once the airflow adjusts to this increase in angle of attack, overall lift is increased, and the aircraft begins to climb. In mathematical terms, this behavior represents a NMP system. To control altitude, a firstorder approximation of the rate of climb $\dot{h}$ is used:

$$
\dot{h}=V \gamma
$$

By prescribing the altitude and total velocity $V$, trajectories $\dot{h}, \gamma$, and $\dot{\gamma}$ can be commanded to the outer loop.

\section{Numerical Simulations}

In this section, trajectory control studies are performed on the proposed controller architecture. The control architecture is initially applied to an elastic aircraft at a heavyweight condition. A simulated climbing and turning trajectory is presented and discussed. The controller is then applied, without changes to any of the gains, to a lightweight condition with the same commanded climbing and turning trajectory. Finally, a representative mission profile segment is developed and simulated.

\section{A. Representative HALE Aircraft}

For the aircraft simulation studies, a representative HALE type aircraft was created, as shown in Fig. $\underline{3}$. The relevant physical properties are summarized in Table 7 . The vehicle was designed to be statically stable for moderate wing deflections in both the

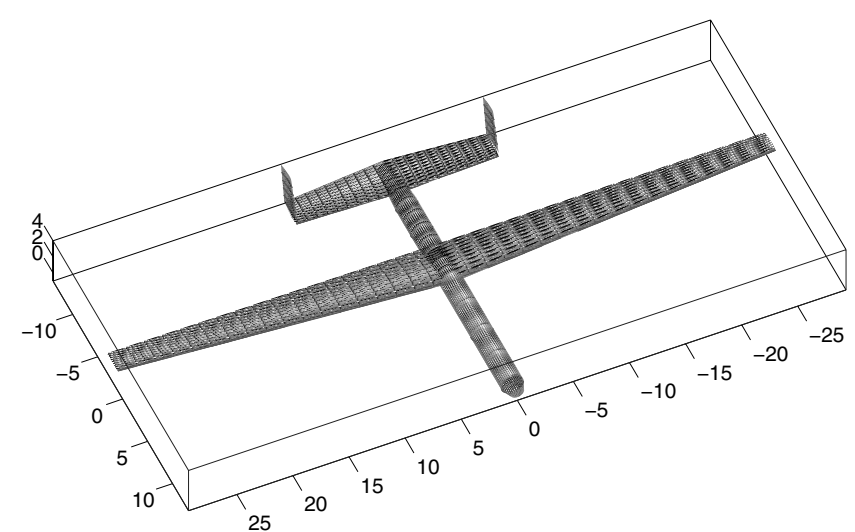

Fig. 3 Representative HALE aircraft model (units are in meters). 
Table 7 Geometric properties of the very flexible aircraft model

\begin{tabular}{|c|c|c|}
\hline \multicolumn{3}{|c|}{ Model parameters } \\
\hline Property & Value & Units \\
\hline Fuselage length & 26.4 & $\mathrm{~m}$ \\
\hline Wing span & 58.6 & $\mathrm{~m}$ \\
\hline Wing area & 196.3 & $\mathrm{~m}^{2}$ \\
\hline Root chord & 4.5 & $\mathrm{~m}$ \\
\hline Tip chord & 2.2 & $\mathrm{~m}$ \\
\hline Aspect ratio & 17.5 & \\
\hline Wing incidence angle & $3.0^{\circ}$ & 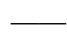 \\
\hline Horizontal tail span & 18.0 & $\mathrm{~m}$ \\
\hline Horizontal root chord & 3.5 & $\mathrm{~m}$ \\
\hline Horizontal tip chord & 2.45 & $\mathrm{~m}$ \\
\hline Horizontal tail incidence angle & $-4.5 \mathrm{deg}$ & 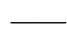 \\
\hline Vertical tail span & 4.0 & $\mathrm{~m}$ \\
\hline Vertical root chord & 2.45 & $\mathrm{~m}$ \\
\hline Vertical tip chord & 2.0 & $\mathrm{~m}$ \\
\hline Wing/horizontal tail airfoil & NACA 4415 & 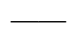 \\
\hline Vertical tail airfoil & NACA 0012 & 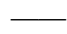 \\
\hline Aileron location & $16.3-22.8$ & $\mathrm{~m}$ \\
\hline Aileron, elevator, rudder chord & $0.2 c_{\text {local }}$ & 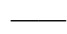 \\
\hline Elevator span location & $1.8-9.0$ & $\mathrm{~m}$ \\
\hline Rudder span location & $0.8-3.2$ & $\mathrm{~m}$ \\
\hline Elements per wing & 9 & - \\
\hline Elements per horizontal tail & 5 & - \\
\hline Elements per vertical tail & 5 & $\ldots$ \\
\hline Elements in fuselage & 10 & 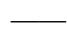 \\
\hline Total no. of elements & 48 & $\longrightarrow$ \\
\hline No. of second-order states & 192 & - \\
\hline No. of first-order states & 241 & {[} \\
\hline
\end{tabular}

longitudinal and lateral axes. Table 8 also includes the trimmed longitudinal controls and state (elevator angle, thrust level, and angle of attack) for both gliding and 1- $g$-level flights. Additional details of the vehicle's mass, structural damping, and stiffness parameters are provided in [33].

The vehicle is a conventional wing/body/tail configuration with twin vertical tails. It is representative of a HALE aircraft concept being considered by the USAF. The aircraft has the conventional control surfaces of elevator, aileron, and twin rudders. The elevator is such that a positive elevator control input $\delta_{e}$ results in a negative pitching moment (nose down). The left and right ailerons have a $-1: 1$ gearing ratio, such that a positive aileron control input $\delta_{a}$ results in a roll to the left (left wing down). The twin rudders have a 1:1 gear ratio such that a positive rudder control input $\delta_{r}$ produces a positive yawing moment (nose left). Recall that the $B$ reference frame orientation is $x$ positive out the right wing, $y$ is positive out the nose, and $z$ is positive up. Thrust is accomplished using a simple point force applied at the origin of the $B$ reference frame and in the $y$ direction, such that a positive thrust input $\delta_{t}$ results in an acceleration in the positive $y$ direction.

\section{B. Representative Flexible Aircraft Trajectory Tracking}

A single controller is presented and was applied to trajectory control for climb only, bank only, and simultaneous climb and bank all at a given (heavy) fuel state. For brevity only, the simultaneous climb and bank results are presented here (see [54] for additional results). The controller is then applied at an alternate (empty) fuel state, demonstrating its robustness to significant mass and inertia changes. Finally, a representative mission profile is simulated.

\section{Flexible Aircraft Controller Design}

The two separate inner-loop controllers for a flexible aircraft were presented in Sec. III.D.1. For the longitudinal motion, a dynamic inversion approach was developed in which the longitudinal states used in development of the controller were longitudinal and vertical velocity $\left(v_{B_{y}}\right.$ and $\left.v_{B_{z}}\right)$ and pitch rate $\omega_{B_{x}}$. The corresponding control inputs were elevator $\delta_{e}$, and throttle $\delta_{t}$. Initially, all three longitudinal states were attempted to be controlled through the use of a pseudoinverse of the resulting control effector matrix function, $[(\partial h / \partial x) g(x)]$. The use of the pseudoinverse resulted in a stable inner-loop longitudinal controller, but it had poor tracking performance. A significant improvement is achieved by only controlling $v_{B_{y}}$ and $\omega_{B_{x}}$. The resulting set of error states are modified from the proposed set [Eq. (5) $]$ to

$$
\dot{e}_{\text {long }}=\left\{\begin{array}{c}
v_{B_{y}}-v_{B_{y} \mathrm{com}} \\
\omega_{B_{x}}-\omega_{B_{x} \mathrm{com}}
\end{array}\right\}
$$

The resulting inner-loop longitudinal state vector is

$$
\chi_{\text {long }}=\left\{\begin{array}{lll}
v_{B_{y}} & \omega_{B_{x}} & e_{\text {long }}
\end{array}\right\}^{T}
$$

A standard LQR controller is applied to the resulting LTI state-space system (see Sec. III for details), in which the weighting matrices are

$$
\begin{gathered}
Q_{\text {long }}=\operatorname{diag}\left[\begin{array}{llll}
1 & 1 & 10^{2} & 10^{4}
\end{array}\right] \\
R_{\text {long }}=\operatorname{diag}\left[\begin{array}{ll}
1 & 1
\end{array}\right]
\end{gathered}
$$

For the lateral inner-loop controller, the error states are also modified from Eq. (93) to

$$
\dot{e}_{\text {lat }}=\left\{\begin{array}{c}
v_{B_{x}}-0 \\
\omega_{B_{y}}-\omega_{B_{y} \mathrm{com}}
\end{array}\right\}
$$

\begin{tabular}{|c|c|c|c|c|c|}
\hline \multicolumn{6}{|c|}{ Model parameters } \\
\hline \multirow[t]{2}{*}{ Property } & \multicolumn{4}{|c|}{ Value } & \multirow[t]{2}{*}{ Units } \\
\hline & Light, no thrust & Heavy, no thrust & Light, thrust for level flight & Heavy, thrust for level flight & \\
\hline Elevator deflection angle $\delta_{e}$ & $4.51 \mathrm{deg}$ & $-16.80 \mathrm{deg}$ & $-6.89 \mathrm{deg}$ & $-13.68 \mathrm{deg}$ & - \\
\hline Thrust required $\delta_{t}$ & 0 & 0 & $3.21 \times 10^{4}$ & $1.12 \times 10^{5}$ & $\mathrm{~N}$ \\
\hline Aircraft angle of attack $\alpha$ & $0.80 \mathrm{deg}$ & $7.62 \mathrm{deg}$ & $1.93 \mathrm{deg}$ & $7.30 \mathrm{deg}$ & 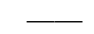 \\
\hline Fuel mass & 0 & 32,000 & 0 & 32,000 & $\mathrm{~kg}$ \\
\hline Total mass & $2.10 \times 10^{4}$ & $5.38 \times 10^{4}$ & $2.10 \times 10^{4}$ & $5.38 \times 10^{4}$ & $\mathrm{~kg}$ \\
\hline Fuel fraction & 0.0 & 59.5 & 0.0 & 59.5 & $\%$ \\
\hline$I_{x x}^{s s}$ & $1.48 \times 10^{6}$ & $1.75 \times 10^{6}$ & $1.49 \times 10^{6}$ & $1.75 \times 10^{6}$ & $\mathrm{~kg} \cdot \mathrm{m}^{2}$ \\
\hline$I_{y y}^{s s}$ & $8.20 \times 10^{5}$ & $2.93 \times 10^{6}$ & $8.19 \times 10^{5}$ & $2.93 \times 10^{6}$ & $\mathrm{~kg} \cdot \mathrm{m}^{2}$ \\
\hline$I_{z z}^{s s}$ & $2.27 \times 10^{6}$ & $4.46 \times 10^{6}$ & $2.26 \times 10^{6}$ & $4.47 \times 10^{6}$ & $\mathrm{~kg} \cdot \mathrm{m}^{2}$ \\
\hline$I_{x y}^{s s}$ & 0 & 0 & 0 & 0 & $\mathrm{~kg} \cdot \mathrm{m}^{2}$ \\
\hline$I_{x z}^{s s}$ & 0 & 0 & 0 & 0 & $\mathrm{~kg} \cdot \mathrm{m}^{2}$ \\
\hline$I_{y z}^{s s}$ & $1.82 \times 10^{4}$ & $9.20 \times 10^{4}$ & $2.06 \times 10^{4}$ & $9.00 \times 10^{4}$ & $\mathrm{~kg} \cdot \mathrm{m}^{2}$ \\
\hline$x_{\text {c.m. }}$ & 0 & 0 & 0 & 0 & $\mathrm{~m}$ \\
\hline$y_{\text {c.m. }}$ & 3.13 & $4.33 \times 10^{-3}$ & 3.14 & $5.64 \times 10^{-3}$ & $\mathrm{~m}$ \\
\hline$z_{\mathrm{c} . \mathrm{m} .}$ & 0.29 & 0.79 & 0.34 & 0.77 & $\mathrm{~m}$ \\
\hline
\end{tabular}

This was done for two reasons. First, because of the addition of the

Table 8 Control and aircraft states and inertia properties of the very flexible aircraft model

${ }^{\mathrm{a}} \mathrm{I}^{s s}$ are the inertia properties in a deformed steady-state configuration. All aircraft simulations are begun at sea-level conditions and $65 \mathrm{~m} / \mathrm{s}$ level flight. 
strain $\epsilon$ and strain-rate $\dot{\epsilon}$ states to the lateral inner-loop state vector [Eq. (63)], it is difficult to find weighting matrices $Q$ and $R$, which provide solutions to the algebraic Ricatti equation of the $L Q R$ technique. Removal of the yaw rate state assisted this problem. Second, it was found that commanding the states of lateral velocity $v_{B_{x}}$ and roll rate $\omega_{B_{y}}$ provided stable tracking of the removed yaw rate $\omega_{B_{z}}$. As described in Sec. III.D.2, an LQR controller is applied to the lateral motion inner-loop states. The weighting matrices are

$$
\begin{gathered}
Q_{\text {lat }}=\operatorname{diag}\left[\begin{array}{ccccc}
Q_{\epsilon} & Q_{v_{B_{x}}} & Q_{\omega_{B_{y}}} & Q_{\omega_{B_{z}}} & Q_{e_{\text {lat }}}
\end{array}\right] \\
R_{\text {lat }}=\operatorname{diag}\left[\begin{array}{ll}
1 & 1
\end{array}\right]
\end{gathered}
$$

where

$$
Q_{\text {lat }}=\operatorname{diag}\left[\begin{array}{llllll}
10^{-3} & 0 & 10 & 0 & \left(10^{2}\right. & \left.10^{3}\right)
\end{array}\right]^{T}
$$

The outer-loop gains, described in Sec. III.C.3, are loosely designed using the guidelines of Ziegler and Nichols $[\underline{44}, \underline{45}]$. The final chosen values are

$$
\begin{array}{r}
k_{\Phi}=0.9 \quad k_{I \Phi}=0.225 \quad k_{d \Phi}=0.45 \\
k_{\dot{\Phi}}=0.9 \quad k_{I \dot{\Phi}}=0.225 \quad k_{I I \dot{\Phi}}=0.0225 \\
k_{\gamma}=0.5 \quad k_{I \gamma}=0.5 \quad k_{I I \gamma}=0 \quad k_{d \gamma}=0.05 \\
k_{\dot{\gamma}}=0.5 \quad k_{I \dot{\gamma}}=0.5 \quad k_{I I \dot{\gamma}}=0.1
\end{array}
$$

Finally, because of the high frequency of numerical errors occurring during the first few time steps of the simulations, a thirdorder low-pass Butterworth filter [55] was applied to the difference equations of flight path angle, $\gamma$, and Euler bank angle, $\Phi$, and their rates given in Eq. (58). The filtered equation for the commanded flight path elevation angle difference is

$$
\dot{x}_{\gamma \mathrm{com}}=A_{f} x_{\gamma \mathrm{com}}+B_{f} \gamma_{\mathrm{com}}\left(\gamma_{\mathrm{com}}\right)_{f}=C_{f} x_{\gamma \mathrm{com}}
$$

For the third-order 2-Hz low-pass Butterworth filter used, the statespace matrices are

$$
A_{f}=\left[\begin{array}{ccc}
-\pi & 0 & 0 \\
\pi & -\pi & -\pi \\
0 & \pi & 0
\end{array}\right] \quad B_{f}=\left\{\begin{array}{l}
\pi \\
0 \\
0
\end{array}\right\} \quad C_{f}=\left[\begin{array}{lll}
0 & 0 & 1
\end{array}\right]
$$

The resulting outer-loop flight path and Euler bank angle commands are modified from Eq. (무) to

$$
\begin{aligned}
& \Delta \gamma_{\text {com }}=k_{\gamma}(\Delta \gamma)_{f}+k_{I \gamma} \int_{0}^{t}(\Delta \gamma) \mathrm{d} \tau \\
& \quad+k_{I I \gamma} \int_{0}^{t}\left(\int_{0}^{t}(\Delta \gamma) \mathrm{d} \tau\right) \mathrm{d} \tau+k_{d \gamma}(\Delta \dot{\gamma})_{f} \\
& \Delta \dot{\gamma}_{\text {com }}=k_{\dot{\gamma}}(\Delta \dot{\gamma})_{f}+k_{I \dot{\gamma}}(\Delta \gamma)_{f}+k_{I I \dot{\gamma}} \int_{0}^{t}(\Delta \gamma) \mathrm{d} \tau \\
& \quad+k_{I I I} \int_{0}^{t}\left(\int_{0}^{t}(\Delta \gamma) \mathrm{d} \tau\right) \mathrm{d} \tau \\
& \Delta \Phi_{\text {com }}=k_{\Phi}(\Delta \Phi)_{f}+k_{I \Phi} \int_{0}^{t}(\Delta \Phi) \mathrm{d} \tau+k_{d \Phi} \Delta \dot{\Phi}_{f} \\
& \Delta \dot{\Phi}_{\text {com }}=k_{\dot{\Phi}}(\Delta \dot{\Phi})_{f}+k_{I \dot{\Phi}}(\Delta \Phi)_{f}+k_{I I \dot{\Phi}} \int_{0}^{t}(\Delta \Phi) \mathrm{d} \tau
\end{aligned}
$$

\section{Climbing and Turning Trajectory}

A combined climbing (100-m altitude change) and turning (20deg bank angle change) trajectory was commanded at a heavyweight fuel condition. The flight path angle, altitude, and bank angle change trajectories are seen in Fig. 4. The overshoot in flight path angle is because of the coupling of the lateral and longitudinal motion. The overshoot in bank angle between 10 and $25 \mathrm{~s}$ is because of an initial lag (because of wing flexibility) between 2 and $5 \mathrm{~s}$, and then an excessive bank angle rate between 5 and $10 \mathrm{~s}$ as the controller tried to compensate for the initial lag. The $B$ reference frame linear and angular velocities are shown in Figs. 5-7. The adverse effects of the strong lateral and longitudinal coupling of this maneuver are diminished because of the overall good control on total velocity [Eq. (27) and Fig. 5 (between 64.8 to $65.2 \mathrm{~m} / \mathrm{s}$ )] and acceptable control on the flight path angle [Fig. 4]. This results in less than a 5-m steady-state error in altitude and excellent steady-state tracking of the Euler bank angle [Figs. 4b and 4c]. For this simulation, elevator deflections are between -19.5 and $-10.5 \mathrm{deg}$, aileron deflections between -5.25 and $3.75 \mathrm{deg}$, and rudder deflection between -19.5 and $-10.5 \mathrm{deg}$.

\section{Banking and Climbing Trajectory at Empty Fuel State}

To investigate the robustness of the proposed controller architecture, the same banking and climbing trajectory of the previous section is repeated here, but at an empty fuel condition. Figure 8 shows the desired and actual response in flight path angle, altitude, and bank angle. Whereas the bank angle response is similar to heavy fuel condition (Fig. 4c), there are greater excursions in the flight path angle and altitude. These excursions are attributed to the outer-loop controller. The $B$ reference frame linear and angular velocity trajectories (actual, commanded, and desired) are shown in Figs. 9-11. An examination of the vertical velocity (Fig. 9b) highlights a problem with applying the controller designed for the heavyweight to the lightweight condition. In the outer-loop nonlinear transformation, Sec. III.C.3, the body angle of attack $\alpha$ is a derived and required parameter, based upon a steady-state wings-level zero

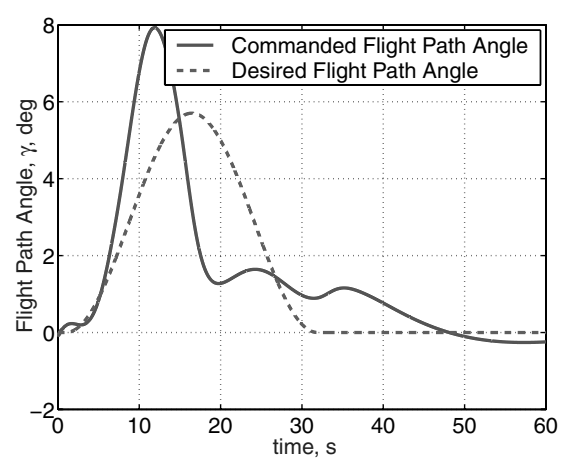

a)

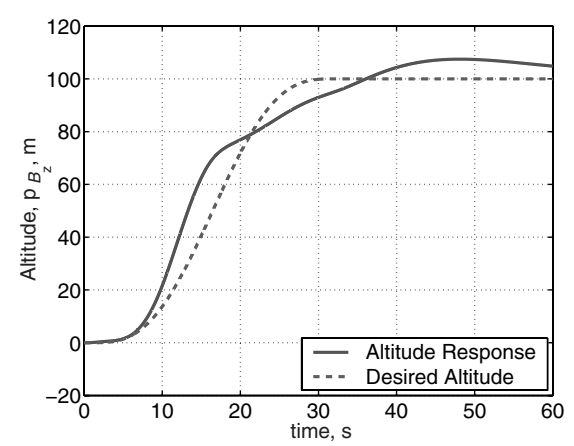

b)

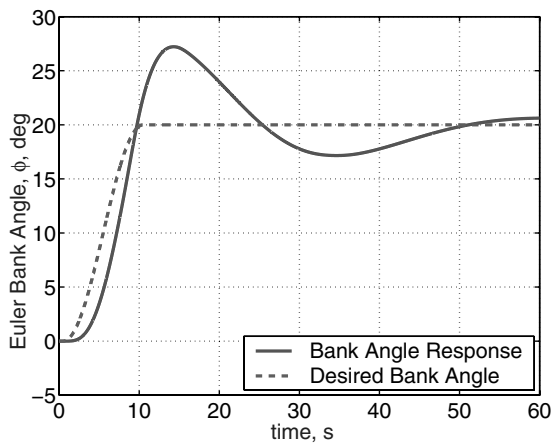

c)

Fig. 4 Commanded a) flight path angle, b) altitude, and c) Euler angle for the heavyweight condition; bank/climb command. 


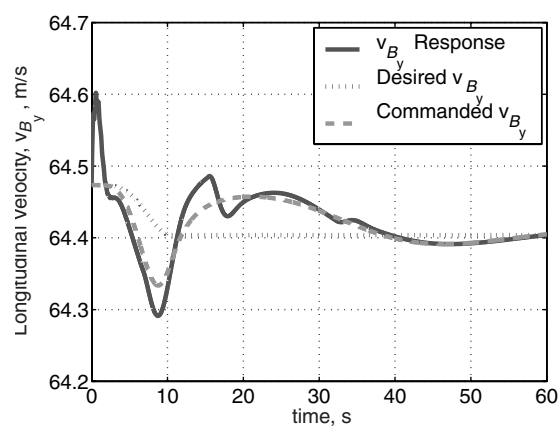

a)

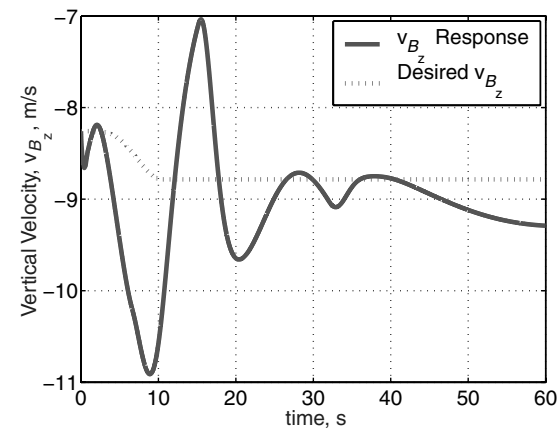

b)

Fig. $5 B$ reference frame velocities, heavyweight condition, and bank/climb command.

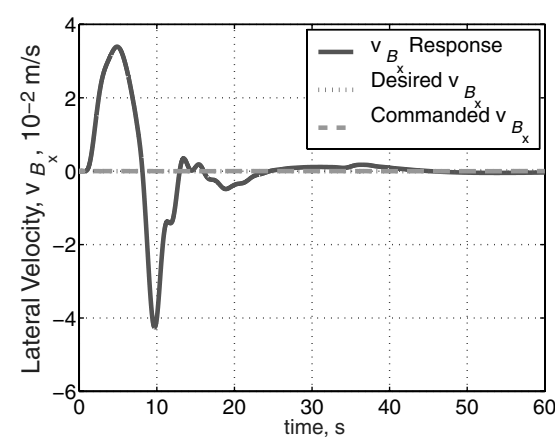

a) Lateral velocity

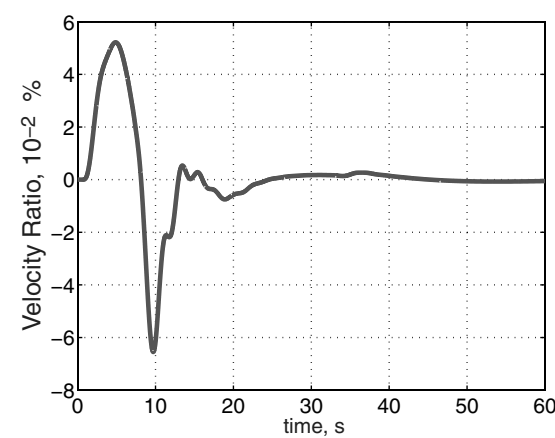

b) Lateral velocity as a percentage of total velocity

Fig. $6 B$ reference frame lateral velocity, heavyweight condition, and bank/climb command.

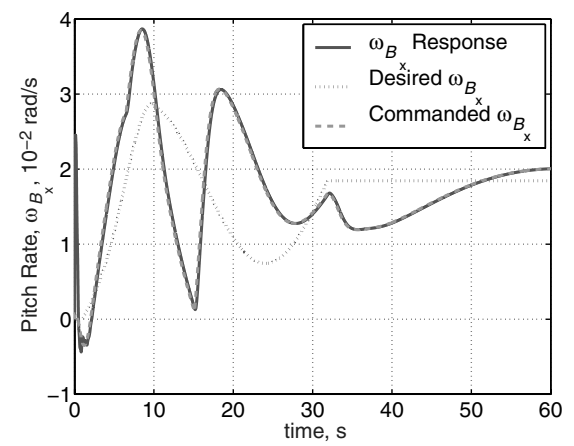

a)

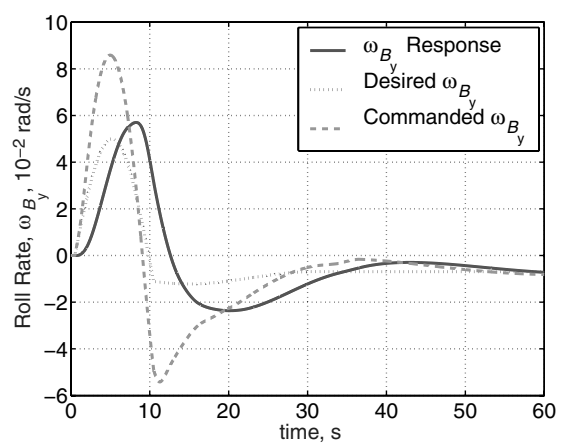

b)

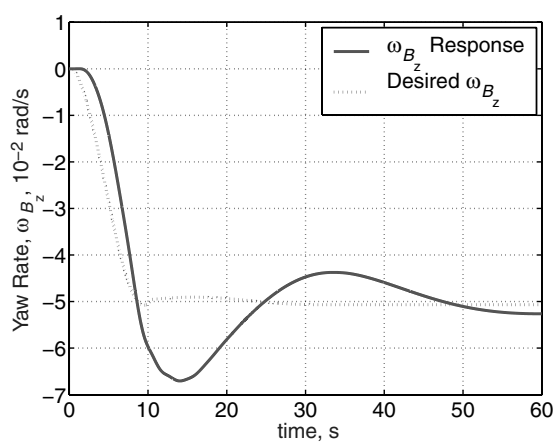

c)

Fig. $7 \quad B$ reference frame angular velocities, heavyweight condition, and bank/climb command.

angle of attack $\alpha_{0}$ given in Eq. (48). The heavyweight controller was designed using a trim body angle of attack $\alpha_{0}$ of $7.30 \mathrm{deg}$, presented in Table $\underline{8}$ and applied to the lightweight condition here. Note, for a lightweight condition, the trim body angle of attack $\alpha_{0}$ is $1.93 \mathrm{deg}$.
Despite the resulting large discrepancy in $\alpha_{0}$ and vertical velocities between the light and heavyweight conditions, the controller performs adequately for the significant change in mass; see Table $\underline{8}$. This is because vertical velocity is not actively controlled, and the

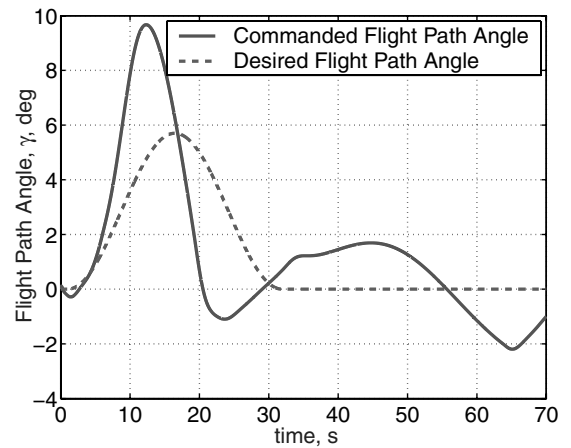

a)

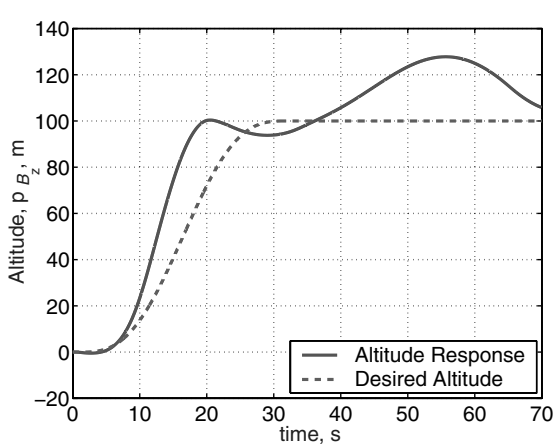

b)

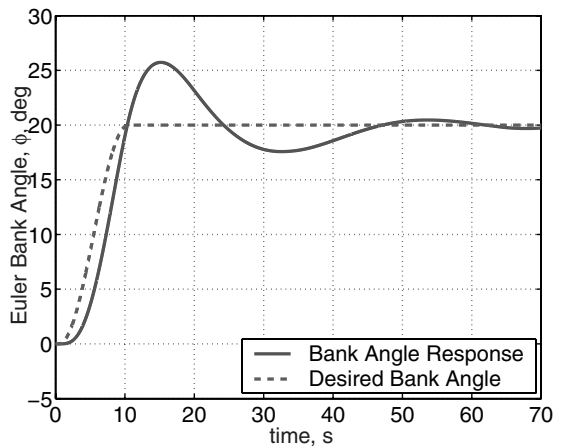

c)

Fig. 8 Commanded a) flight path angle, b) altitude, and c) Euler angle for lightweight condition; bank/climb command. 


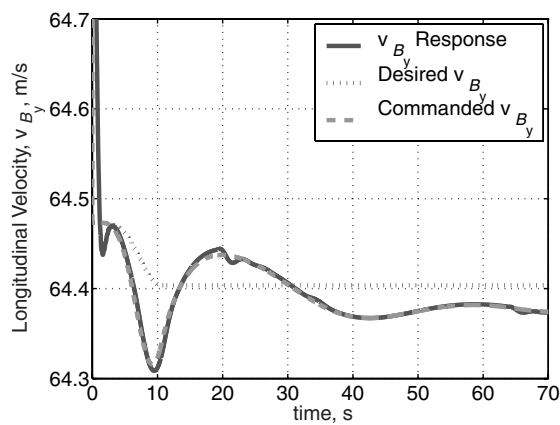

a)

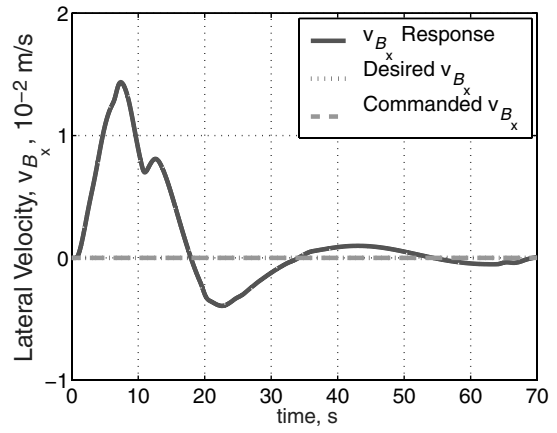

a) Lateral velocity

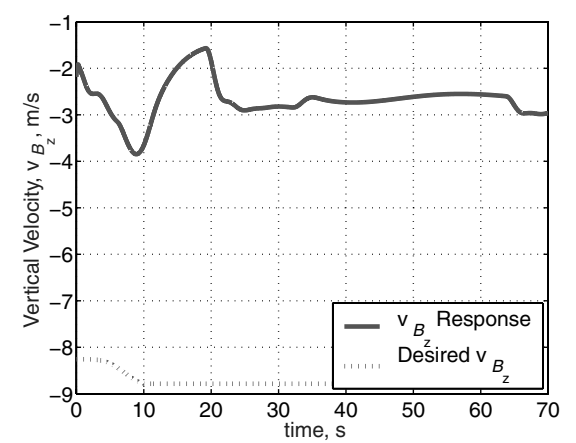

b)

Fig. $9 B$ reference frame velocities, lightweight condition, and bank/climb command.

$\begin{array}{ll}\text { Fig. } 10 & B \text { reference frame lateral velocity, heavyweight condition, and bank/climb command. }\end{array}$

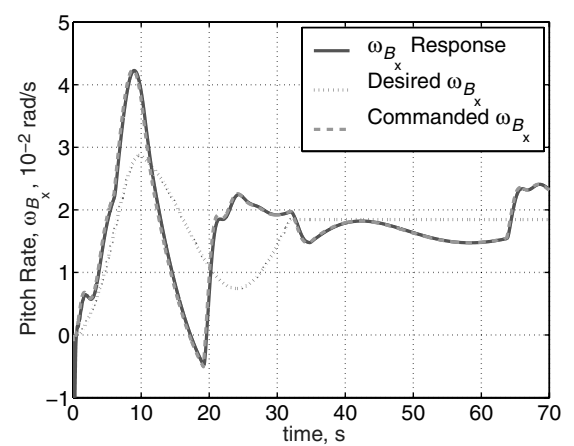

a)

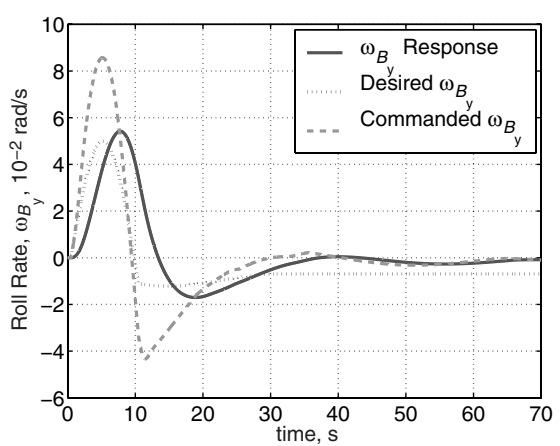

b)

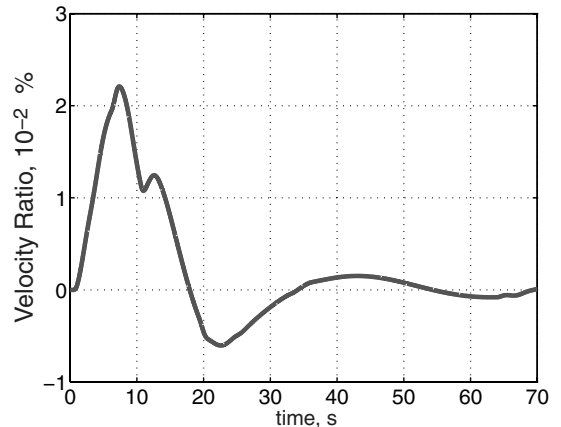

b) Lateral velocity as a percentage of total velocity

Fig. $11 B$ reference frame angular velocities, lightweight condition, and bank/climb command.

other $B$ reference frame velocities are not as significantly affected by $\alpha_{0}$. For this simulation, elevator deflections are between -14 and $-9.5 \mathrm{deg}$, aileron deflections between -5 and $2.5 \mathrm{deg}$, and rudder deflection between -1.2 and $0 \mathrm{deg}$.

\section{Mission Profile Segment Case}

A representative mission profile segment simulation is presented here. The aircraft is commanded to perform the following sequence of maneuvers: climb and turn, roll out of turn and continue climbing, level off, level turn, descent and turn, roll out of turn and continue descending, and level off as seen in Fig. 12. The altitude and associated flight path angle along with the Euler bank angle desired and actual trajectories are seen in Figs. 13 and 14. Altitude tracking presents a maximum overshoot of $46 \overline{\mathrm{m}}$ following the commanded climb. This overshoot occurs while the bank angle is settling to its commanded 0-deg angle. The overshoot in altitude is acceptable given that this class of aircraft is expected to fly in the National Airspace System. Typical piloted aircraft flying under instrument

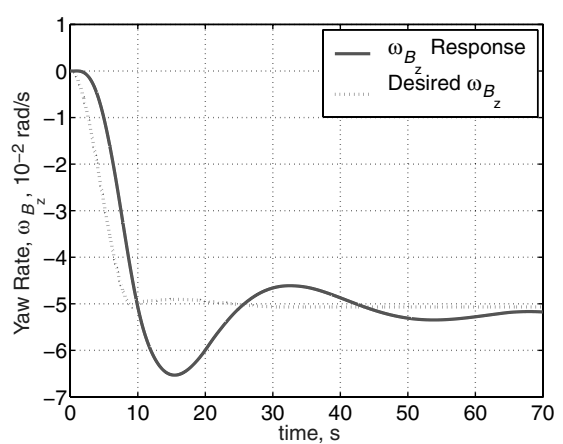

c) 


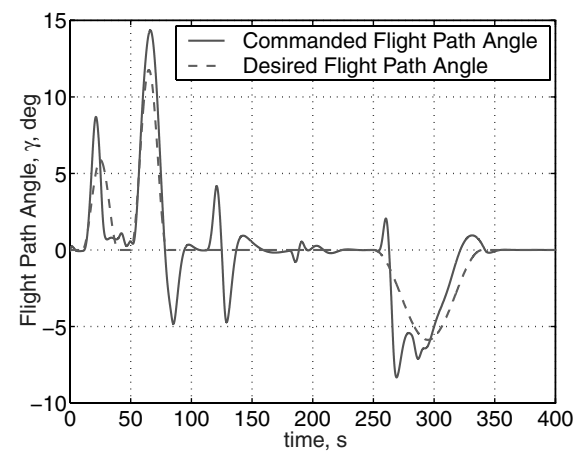

a)

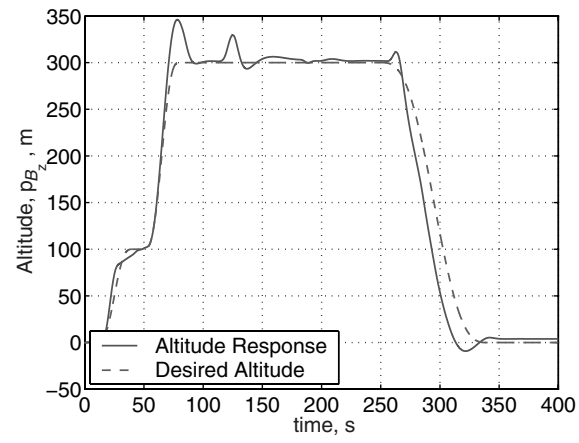

b)

Fig. 13 Commanded a) flight path angle and b) altitude for the heavyweight condition.

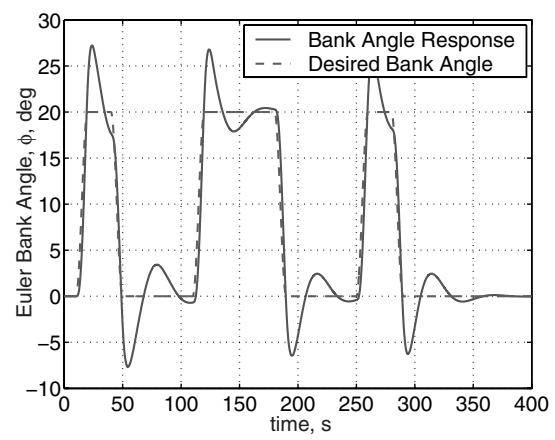

Fig. 14 Commanded Euler bank angle for the heavyweight condition.

flight rules (IFR) are required to maintain less than about $30-\mathrm{m}$ steady-state altitude deviations and are allowed up to 60-m temporary deviations. The corresponding longitudinal and lateral control inputs are seen in Fig. 15. During the second portion of the commanded climb, an increase in throttle and decrease in elevator are seen (between 50 and $75 \mathrm{~s}$ ) corresponding to the greater commanded climb rate $(6.67 \mathrm{~m} / \mathrm{s}$ at $25 \mathrm{~s}$ and $13.33 \mathrm{~m} / \mathrm{s}$ at $65 \mathrm{~s})$. There is a steadystate altitude error of less than $2 \mathrm{~m}$ at $250 \mathrm{~s}$ and $4 \mathrm{~m}$ at $400 \mathrm{~s}$. Bank angle overshoots are less than $7.2 \mathrm{deg}$ with excellent steady-state tracking. The $B$ reference frame desired, commanded, and actual velocities are seen in Figs. 16-18. There is excellent tracking of the commanded longitudinal velocity (Fig. 16b) and pitch rate (Fig. 18a). Tracking error between the desired and commanded values in the longitudinal motion are attributed to outer-loop controller architecture. For the lateral motion, the sideslip velocity (Fig. 16a) is seen to be below $0.04 \mathrm{~m} / \mathrm{s}$ or $0.05 \mathrm{deg}$ of the total velocity, $V$. Additionally, the roll rate (Fig. 18b) has acceptable tracking, and the yaw rate (Fig. 18c), although not directly controlled, has relatively good and stable tracking of the desired trajectory.

\section{Conclusions}

This paper presented a proposed two-level control architecture to address coupled nonlinear flight dynamics and nonlinear aeroelastic responses found in very flexible aircraft. The inner- and outer-loop architecture demonstrated an effective means for accomplishing trajectory control of this class of aircraft. Although more complex than previously developed linear aeroelastic controllers, the proposed architecture was necessary to achieve stable tracking as compared with traditional linear methods. Furthermore, the
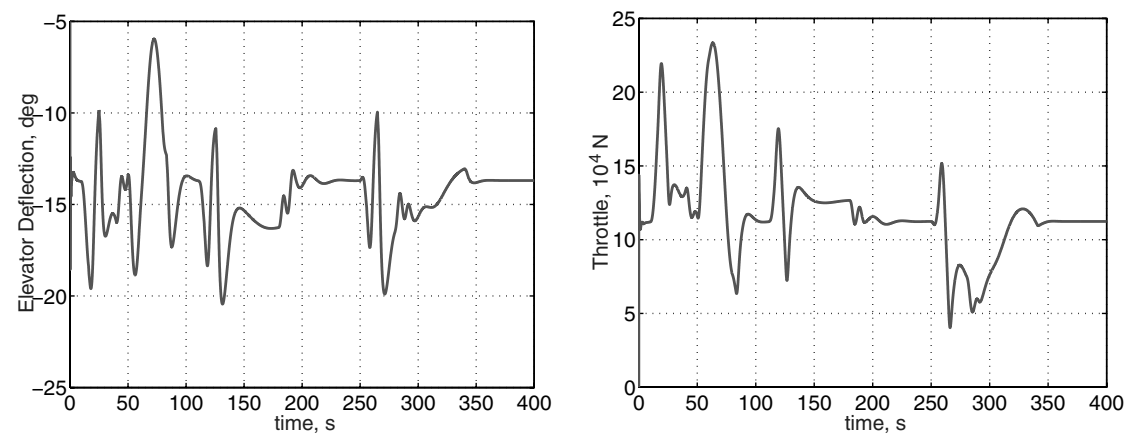

a)

b)
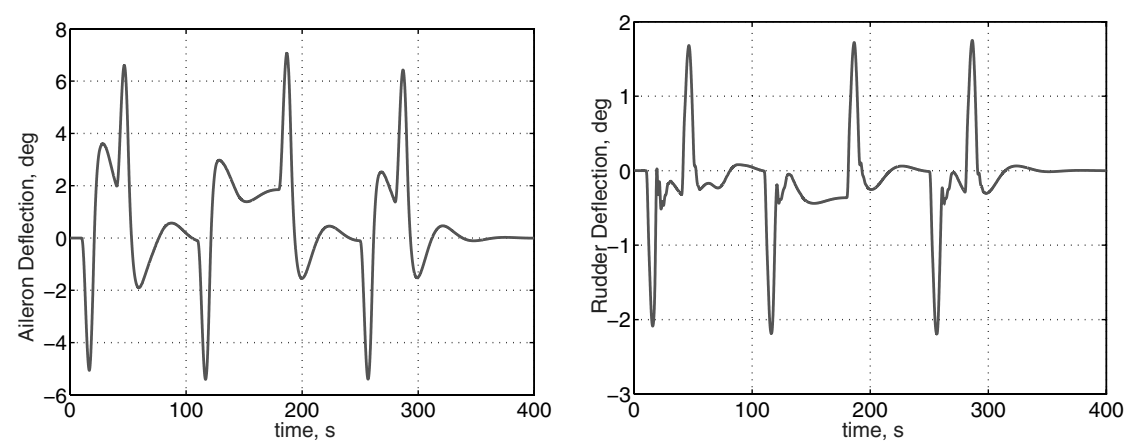

d)

c)

Fig. 15 Longitudinal and lateral flight controls for the heavyweight condition. 


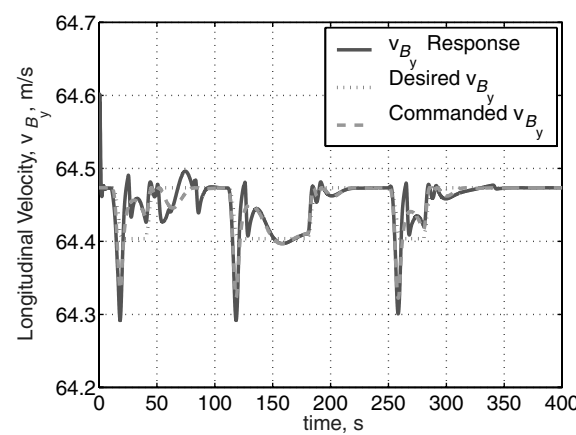

a)

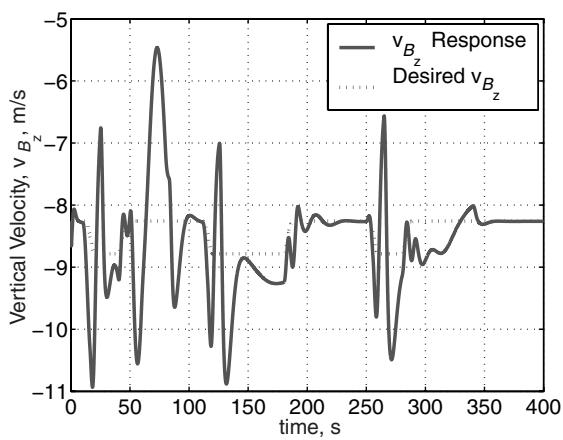

b)

Fig. $16 B$ reference frame velocities for the heavyweight condition.

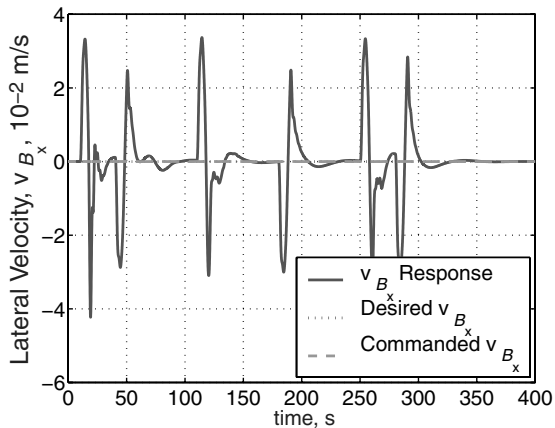

a) Lateral velocity

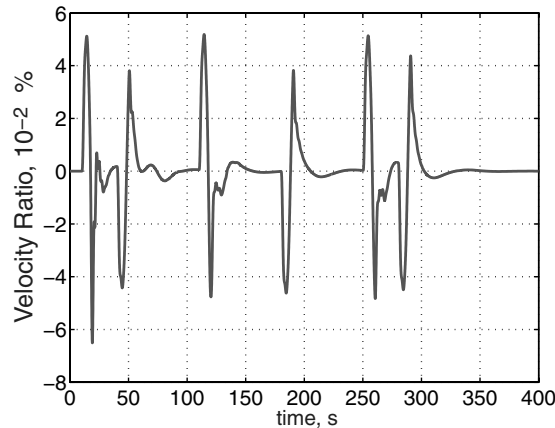

b) Lateral velocity as a percentage of total velocity

Fig. $17 B$ reference frame lateral velocity for the heavyweight condition.

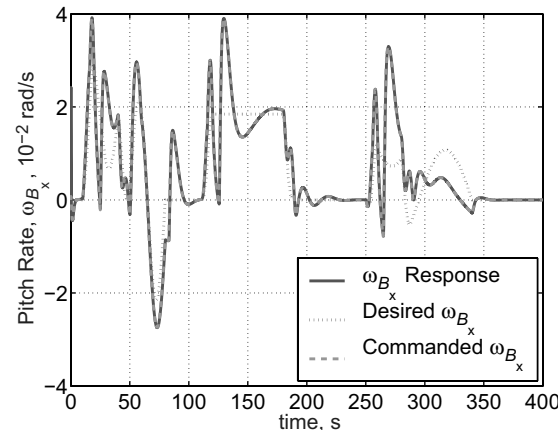

a) Pitch rate

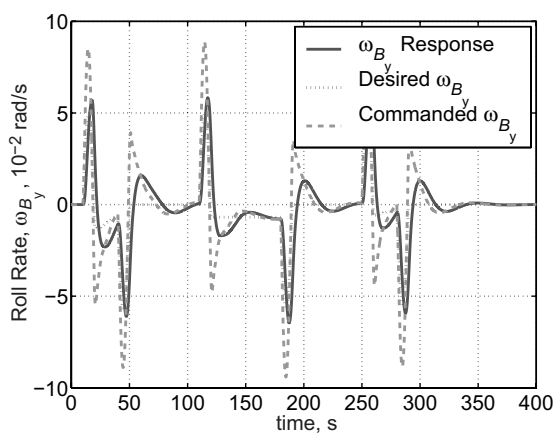

b) Roll rate

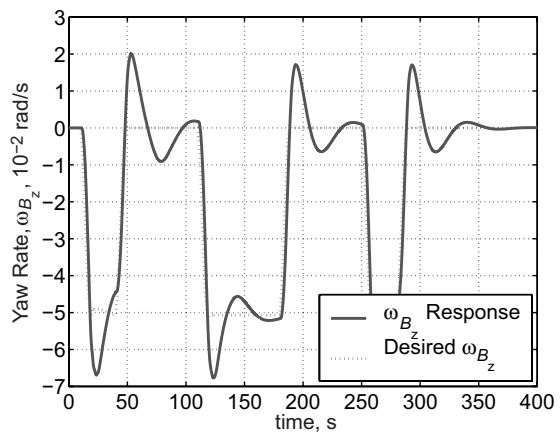

c) Yaw rate

Fig. $18 B$ reference frame angular velocities for the heavyweight condition.

separation of longitudinal and lateral motion control handled by separate controllers proved to be very effective. Finally, although not presented here, the general trends of increased integral gain destabilizing system response and increased derivative gain stabilizing and slowing system response were seen in the development of the outer-loop nonlinear gains. The control architecture was shown numerically to track altitude and bank angle changes in the presence of smooth air, full elastic state feedback, and perfect sensors. The purpose of these studies was not to design a perfect controller, but rather to demonstrate the viability of the proposed method. In such, there is more tuning that could be accomplished for tighter trajectory control.

\section{Acknowledgment}

The views expressed in this article are those of the authors and do not reflect the official policy or position of the U.S. Air Force, Department of Defense, or the U.S. government.

\section{References}

[1] Tilmann, C. P., Flick, P. M., Martin, C. A., and Love, M. H., "HighAltitude Long-Endurance Technologies for Sensor Craft," NATO Paper MP-104-P-26-1, 7-10 Apr. 2003.

[2] Whitson, S., "The Proteus, Giving Shape to Forms Unknown," Private Pilot, Vol. 33, No. 12, Dec. 1998, pp. 44-50.

[3] Noll, T. E., Brown, J. M., Perez-Davis, M. E., Ishmael, S. D., Tiffany, G. C., and Gaier, M., "Investigation of the Helios Prototype Aircraft Mishap," Vol. 1, NASA, Jan. 2004.

[4] Anon., "Unmanned Aircraft Systems Roadmap 2005-2030," U.S. Dept. of Defense, Office of the Secretary of Defense, 4 Aug. 2005.

[5] Schroer, R., "Stability and Control [A Century of Powered Flight 1903 2003]," IEEE Aerospace and Electronic Systems Magazine, Vol. 18, No. 7, July 2003, pp. 37-42.

[6] Nelson, R. C., Flight Stability and Automatic Control, 2nd ed., Princeton Univ. Press, Princeton, NJ, 1998, p. 281.

[7] Mukhopadhyay, V., "Historical Perspective on Analysis and Control of Aeroelastic Responses," Journal of Guidance, Control, and Dynamics, Vol. 26, No. 5, Sept.-Oct. 2003, pp. 673-684.

[8] Newman, B., and Buttrill, C., "Conventional Flight Control for an 
Aeroelastic Relaxed Static Stability High-Speed Transport," AIAA Guidance, Navigation, and Control Conference, 95-3250-CP, AIAA, Reston, VA, 7-10 Aug. 1995, pp. 717-726.

[9] Tuzcu, I., "Dynamics and Control of Flexible Aircraft," Ph.D. Dissertation, Virgina Polytechnic Ins. and State Univ., Blacksburg, VA, Dec. 2001

[10] Meirovitch, L., and Tuzcu, I., "Unified Theory for the Dynamics and Control of Maneuvering Flexible Aircraft," AIAA Journal, Vol. 42, No. 4, Apr. 2004, pp. 714-727.

[11] Pedro, J. O., and Bigg, C. G., "Development of a Flexible Embedded Aircraft/Control System Simulation Facility," AIAA Paper 2005-5889, 15-18 Aug. 2005.

[12] Cesnik, C. E. S., and Ortega-Morales, M., "Active Aeroelastic Tailoring of Slender Flexible Wings," International Forum on Aeroelasticity and Structural Dynamics, Council of European Aerospace Societies, Madrid, 5-7 June 2001.

[13] Chavez, F. R., and Schimdt, D. K., "Systems Approach to Characterizing Aircraft Aeroelastic Model Variation for Robust Control Applications," AIAA Paper 2001-4020, 6-9 Aug. 2001.

[14] Kron, A., deLafontaine, J., and Alazard, D., "Robust 2-DOF H-Infinity Controller for Highly Flexible Aircraft: Design Methodology and Numerical Results," Canadian Aeronautics and Space Journal, Vol. 49, No. 1, Mar. 2003, pp. 19-29.

[15] Li, X., and Agarwal, R. K., "Application of Reduced-Order Models to Robust Control of the Dynamics of a Flexible Aircraft," AIAA Paper 2003-5504, 11-14 Aug. 2003.

[16] Goman, M., Sidoryuky, M., and Ustinovz, A., "Control Law Design for Flexible Aircraft: Comparison of the H1-Based and Classical Methods," AIAA Paper 2005-6265, 15-18 Aug. 2005.

[17] Dardenne, I., and Ferreres, G., "Design of a Flight Control System for a Highly Flexible Aircraft Using Convex Synthesis," 21st Congress of the International Council of the Aeronautical Sciences, International Council of the Aeronautical Sciences Paper 98-1,5,1, 13-18 Sept. 1998.

[18] Patil, M., "Nonlinear Aeroelastic Analysis, Flight Dynamics, and Control of a Complete Aircraft," Ph.D. Dissertation, Georgia Inst. of Technology, Atlanta, May 1999

[19] Patil, M. J., and Hodges, D. H., "Output Feedback Control of the Nonlinear Aeroelastic Response of a Slender Wing," Journal of Guidance, Control, and Dynamics, Vol. 25, No. 2, Mar.-Apr. 2002, pp. 302-308.

[20] Calise, A. J., Kim, N., and Buffington, J. M., "Adaptive Compensation for Flexible Dynamics," AIAA Paper 2002-4917, 5-8 Aug. 2002.

[21] Krishnaswamy, K., and Bugajski, D., "Inversion Based Multibody Control-Launch Vehicle with Fuel Slosh,” AIAA Paper 2005-6149, 15-18 Aug. 2005.

[22] Gregory, I. M., "Dynamic Inversion to Control Large Flexible Transport Aircraft," AIAA Guidance, Navigation, and Control Conference and Exhibit, AIAA, Reston, VA, 10-12 Aug. 1998, pp. 1224-1232; also AIAA Paper 98-4832, 1998

[23] Gregory, I. M., "Stability Result for Dynamic Inversion Devised to Control Large Flexible Aircraft," AIAA Paper 2001-4284, 69 Aug. 2001

[24] Gregory, I. M., "Modified Dynamic Inversion to Control Large Flexible Transport Aircraft: What's Going On?," AIAA Guidance, Navigation, and Control Conference and Exhibit, 99-3998,AIAA, Reston, VA, 911 Aug. 1999, pp. 392-402.

[25] Gregory, I. M., "Design and Stability Analysis of an Integrated Controller for Highly Flexible Advanced Aircraft Utilizing the Novel Nonlinear Dynamic Inversion," Ph.D. Dissertation, California Inst. of Technology, Pasadena, CA, 2005.

[26] Stevens, B. L., and Lewis, F. L., Aircraft Control and Simulation, Wiley, New York, 1992, pp. 62-63, 81, 132-134.

[27] Phillips, W. F., Hailey, C. E., and Gebert, G. A., "A Review of Attitude Kinematics for Aircraft Flight Simulation," AIAA Paper 2000-4302, 14-17 Aug. 2000.

[28] Peters, D., and Johnson, M. J., "Finite-State Airloads for Deformable Airfoils on Fixed and Rotating Wings," Aeroelasticity and Fluid/ Structure Interaction, Proceedings of the Winter Annual Meeting American Society of Mechanical Engineers, Fairfield, NJ, 611 Nov. 1994.

[29] Peters, D. A., and Cao, W., "Finite State Induced Flow Models Part 1: Two-Dimensional Thin Airfoil," Journal of Aircraft, Vol. 32, No. 2, Mar--Apr. 1995, pp. 313-322.

[30] Cesnik, C. E. S., and Brown, E. L., "Active Wing Warping Control of a Joined-Wing Airplane Configuration," AIAA Paper 2003-1715, 7 10 Apr. 2003.

[31] Brown, E. L., "Integrated Strain Actuation in Aircraft with Highly
Flexible Composite Wings,” Ph.D. Dissertation, Massachusetts Inst. of Technology, Boston, June 2003.

[32] Shearer, C. M., and Cesnik, C. E. S., "Nonlinear Flight Dynamics of Very Flexible Aircraft," Journal of Aircraft, Vol. 44, No. 5, Sept.Oct. 2007, pp. 1528-1545; also AIAA Paper 2005-5805, 2005.

[33] Shearer, C. M., "Coupled Nonlinear Flight Dynamics, Aeroelasticity, and Control of Very Flexible Aircraft," Ph.D. Dissertation, Univ. of Michigan, Ann Arbor, MI, 2006.

[34] Arora, J. S., Introduction to Optimum Design, McGraw-Hill, New York, 1989 , pp. 578-580

[35] Shearer, C. M., and Cesnik, C. E. S., "Modified Generalized- $\alpha$ Method for Integrating Governing Equations of Very Flexible Aircraft," AIAA Paper 2006-1747, 1-4 May 2006.

[36] Jansen, K. E., Whiting, C. H., and Hulbert, G. M., "A Generalized- $\alpha$ Method for Integrating the Filtered Navier-Stokes Equations with a Stabilized Finite Element Method," Computer Methods in Applied Mechanics and Engineering, Vol. 190, Nos. 3-4, Oct. 2000, pp. 305319.

[37] Chung, J., and Hulbert, G. M., "A Time Integration Algorithm for Structural Dynamics with Improved Numerical Dissipation: The Generalized- $\alpha$ Method," Journal of Applied Mechanics, Vol. 60, June 1993, pp. 371-375.

[38] Anon., "Flying Qualities of Piloted Vehicles," Military Standard, U.S. Department of Defense, 1987.

[39] Stengel, R. F., Flight Dynamics, Princeton Univ. Press, Princeton, NJ, 2004, pp. 53-56, 159-161.

[40] Heffley, R. K., Hanson, G. D., Jewell, W. F., and Clement, W. F. "Analysis of Pilot Control Strategy," NASA, CR 170399, Apr. 1983.

[41] Heffley, R. K., and Schulman, T. M., "Derivation of Human Pilot Control Laws Based on Literal Interpretation of Pilot Training Literature," AIAA Paper 1981-1822, 19-21 Aug. 1981.

[42] Franklin, G. F., Powell, J. D., and Emami-Naeini, A., Feedback Control of Dynamic Systems, 3rd ed., Addison-Wesley Longman, Reading, MA, 1994, pp. 185-186.

[43] Anderson, J. D., Introduction to Flight, 2nd ed., McGraw-Hill, New York, 1985, pp. 314-316.

[44] Ziegler, J. G., and Nichols, N. B., "Optimum Settings for Automatic Controllers," Transactions of the ASME, Vol. 64, No. 8, Nov. 1942, pp. $759-768$.

[45] Ziegler, J. G., and Nichols, N. B., "Process Lags in Automatic-Control Circuits," Transactions of the ASME, Vol. 65, No. 5, July 1943, pp. 433-444.

[46] Bugajski, D. J., and Enns, D. F., "Nonlinear Control Law with Application to High Angle-of-Attack Flight," Journal of Guidance, Control, and Dynamics, Vol. 15, No. 3, May-June 1992, pp. 761-767.

[47] Adams, R. J., Buffington, J. M., and Banda, S. S., "Design of Nonlinear Control Laws for High Angle-of-Attack Flight," Journal of Guidance, Control, and Dynamics, Vol. 17, No. 4, July-Aug. 1994, pp. 737-746.

[48] Snell, S. A., Enns, D. F., and Garrard, W. L., Jr., "Nonlinear Inversion Flight Control for a Supermaneuverable Aircraft," Journal of Guidance, Control, and Dynamics, Vol. 15, No. 4, July-Aug. 1992, pp. 976-984.

[49] Al-Hiddabi, S. A., "Position Tracking and Path Following for Flight Vehicles Using Nonlinear Control," Ph.D. Dissertation, Univ. of Michigan, Ann Arbor, MI, 2000.

[50] Al-Hiddabi, S. A., and McClamroch, N. H., "Tracking and Maneuver Regulation Control for Nonlinear Nonminimum Phase Systems: Application to Flight Control," IEEE Transactions on Control Systems Technology, Vol. 10, No. 6, Nov. 2002, pp. 780-792. doi:10.1109/TCST.2002.804120

[51] Bernstein, D. S., Matrix Mathematics: Theory, Facts, and Formulas with Application to Linear Systems Theory, 1st ed., Princeton Univ. Press, Princeton, NJ, 2005, p. 72.

[52] Barrett, M., Blue, P., Bugajski, D., Enns, D., Hendrick, R., Jackson, M., Morton, B., Stein, G., Bessollo, J., Virnig, J., Walker, G., Colgren, R., Tait, P., and Smith, R., "Application of Multivariable Control Theory to Aircraft Control Laws," Final Rept. WL-TR-96-3099, Flight Dynamics Directorate, Wright Lab., Wright-Patterson AFB, OH, May 1996

[53] Ito, D., Georgie, J., Valasek, J., and Ward, D. T., "Re-Entry Vehicle Flight Controls Design Guidelines: Dynamic Inversion," Flight Simulation Lab., Texas Engineering Experiment Station, TR NAG91085, Texas A\&M Univ., College Station, TX, May 2001.

[54] Shearer, C. M., and Cesnik, C. E. S., "Trajectory Control for Very Flexible Aircraft," AIAA Paper 2006-6316, 21-24 Aug. 2006.

[55] Freudenberg, J., AERO 580 Linear Feedback Notes, Univ. of Michigan, Ann Arbor, MI, 2004 